\title{
NMD is essential for hematopoietic stem and progenitor cells and for eliminating by-products of programmed DNA rearrangements
}

\author{
Joachim Weischenfeldt, ${ }^{1,2,3}$ Inge Damgaard, ${ }^{1,2,3}$ David Bryder, ${ }^{4}$ Kim Theilgaard-Mönch, ${ }^{1,2,3}$ \\ Lina A. Thoren, ${ }^{5}$ Finn Cilius Nielsen, ${ }^{2}$ Sten Eirik W. Jacobsen, ${ }^{5}$ Claus Nerlov, ${ }^{5,6}$ and \\ Bo Torben Porse $1,2,3,7$ \\ ${ }^{1}$ Section for Gene Therapy Research, Copenhagen University Hospital, 2100 Copenhagen, Denmark; ${ }^{2}$ Department of \\ Clinical Biochemistry, Copenhagen University Hospital, 2100 Copenhagen, Denmark; ${ }^{3}$ Biotech Research and Innovation \\ Centre (BRIC), University of Copenhagen, 2200 Copenhagen, Denmark; ${ }^{4}$ Institute for Experimental Medical Science, \\ BMCI13, Lund University, 22184 Lund, Sweden; ${ }^{5}$ Hematopoietic Stem Cell Laboratory, Lund Strategic Research Centre for \\ Stem Cell Biology and Therapy, Lund University, 22184 Lund, Sweden; ${ }^{6}$ European Molecular Biology Laboratory (EMBL) \\ Mouse Biology Unit, 00015 Monterotondo, Italy
}

\begin{abstract}
Nonsense-mediated mRNA decay (NMD) is a post-transcriptional surveillance process that eliminates mRNAs containing premature termination codons (PTCs). NMD has been hypothesized to impact on several aspects of cellular function; however, its importance in the context of a mammalian organism has not been addressed in detail. Here we use mouse genetics to demonstrate that hematopoietic-specific deletion of Upf2, a core NMD factor, led to the rapid, complete, and lasting cell-autonomous extinction of all hematopoietic stem and progenitor populations. In contrast, more differentiated cells were only mildly affected in Upf2-null mice, suggesting that NMD is mainly essential for proliferating cells. Furthermore, we show that UPF2 loss resulted in the accumulation of nonproductive rearrangement by-products from the Tcrb locus and that this, as opposed to the general loss of NMD, was particularly detrimental to developing T-cells. At the molecular level, gene expression analysis showed that $U p f 2$ deletion led to a profound skewing toward up-regulated mRNAs, highly enriched in transcripts derived from processed pseudogenes, and that NMD impacts on regulated alternative splicing events. Collectively, our data demonstrate a unique requirement of NMD for organismal survival.
\end{abstract}

[Keywords: Hematopoietic stem and progenitor cells; T-cell development; nonsense-mediated mRNA decay; programmed DNA rearrangements; alternative splicing; pseudogenes]

Supplemental material is available at http://www.genesdev.org.

Received December 21, 2007; revised version accepted March 20, 2008.

Nonsense-mediated mRNA decay (NMD) is part of a larger network of RNA surveillance pathways, which ensure that only mRNAs with proper coding potential become available for protein synthesis. Specifically, the NMD machinery recognizes mRNAs with premature termination codons (PTCs) and mediates their degradation. If left available for translation, PTC-containing $\left(\mathrm{PTC}^{+}\right)$mRNAs pose a latent threat to the cell as they may encode proteins with potential dominant-negative properties.

The NMD pathway was initially considered as a guardian against newly arising nonsense mutations ei-

${ }^{7}$ Corresponding author.

E-MAIL porse@rh.dk; FAX 45-3532-5669.

Article is online at http://www.genesdev.org/cgi/doi/10.1101/gad.468808. ther at the DNA level or as a consequence of erroneous transcription or mRNA processing (for recent reviews, see Conti and Izaurralde 2005; Isken and Maquat 2007). More recently, global expression profiling of tissue culture cells subjected to siRNA-mediated knockdown of key NMD components demonstrated that even nonmutated genes are regulated by NMD, suggesting that the NMD pathway actively participates in regulation of normal gene expression (Mendell et al. 2004; Rehwinkel et al. 2005; Wittmann et al. 2006). Of particular importance are the T-cell receptor (TCR) and immunoglobulin (Ig) genes, which undergo programmed DNA rearrangements. Here, two-thirds of the $\mathrm{V}(\mathrm{D}) \mathrm{J}$ recombination events are predicted to be nonproductive by generating a PTC, and the resulting $\mathrm{PTC}^{+}$transcripts were shown to be stabilized both by inhibition of protein synthesis and 
by knockdown of known NMD components (at least in the context of $\mathrm{PTC}^{+}$reporter constructs), thereby strongly suggesting that their clearances are facilitated by NMD (Carter et al. 1995; Li and Wilkinson 1998; Wang et al. 2002). Finally, the NMD pathway has also been proposed to mute genomic noise arising from transcription of pseudogenes and transposable elements as well as retroviruses (Mitrovich and Anderson 2005; Weischenfeldt et al. 2005).

The process of NMD is conserved throughout evolution and several of the mammalian NMD factors, including the core factors UPF1, UPF2, and UPF3A/B have conserved orthologs in lower eukaryotes (for review, see Chang et al. 2007). Depletion or mutation of the UPF proteins abolishes NMD in all eukaryotes studied, and the UPFs are considered to constitute the functional core of the NMD machinery (Perlick et al. 1996; LykkeAndersen et al. 2000). In mammalian cells, PTCs are distinguished from stop codons by their position upstream of exon-exon junctions, which are marked during splicing by the deposition of the exon junction complex (EJC). When a ribosome is stalled on a PTC, UPF1 is recruited together with release factors (eRF1 and eRF3) and SMG-1 (Kashima et al. 2006). If an EJC complex is placed downstream from the PTC, UPF2 acts as a molecular bridge between the stalled ribosome and the EJC, through its interaction with UPF1 and UPF3A/B, respectively (Czaplinski et al. 1998; Singh et al. 2007). Subsequent recruitment of additional NMD components including SMG-5 and SMG-7 leads, through a series of UPF1 phosphorylation and dephosphorylation events, to the disruption of the stalled ribosome-mRNA complex and finally to degradation of the $\mathrm{PTC}^{+}$mRNA in cytoplasmic processing bodies (P-bodies) (Fukuhara et al. 2005; Chang et al. 2007).

In addition to the classical linear NMD pathway, there are also data supporting UPF2-independent and UPF3independent alternative branches of the NMD pathway (Gehring et al. 2005; Chan et al. 2007).

To establish the importance of the UPF proteins for the viability of mammalian cells, these factors have been depleted individually using RNAi-mediated gene silencing. Whereas depletion of UPF2 in human tissue culture had no effect on cellular proliferation, loss of UPF1 resulted in cell cycle arrest and activation of the DNA damage response pathways (Azzalin and Lingner 2006b). In the mouse, Upf1 knockout led to early embryonic lethality, and attempts to generate embryonic stem (ES) cell lines from Upf1-null blastocysts were unsuccessful (Medghalchi et al. 2001). Loss-of-function studies have also been performed in lower eukaryotes including yeast, Caenorhabditis elegans, and Drosophila melanogaster (Pulak and Anderson 1993; He et al. 1997; Metzstein and Krasnow 2006). In the latter organism, both Upf1 and Upf2 were found to be essential for larval viability, a finding that is corroborated by the cell cycle arrest installed by depletion of either UPF1 or UPF2 in Drosophila Schneider cells (Rehwinkel et al. 2005). In contrast, depletion of any of the UPF proteins had no major impact on cell viability in either yeast or C. elegans.
These studies suggest that NMD factors have gained increased importance during the course of evolution, either due to the acquisition of additional functions or due to a change in the repertoire of regulated transcripts toward a more prominent role in post-transcriptional gene regulation.

The hematopoietic system is an attractive experimental model to address issues concerning stem cell function, lineage commitment, and responses to external stimuli. At the apex of the hematopoietic hierarchy resides the long-term hematopoietic stem cell (LT-HSC), a rare cell type present in the bone marrow (BM). In addition to their unique ability to self-renew throughout life, these cells give rise to all the cells of the blood system, through a series of defined differentiation steps. The capacity to specifically identify, isolate, and characterize LT-HSCs, their immediate multipotent descendants, and downstream lineage-committed progenitors has yielded unprecedented insights into issues of stem cell self-renewal, lineage relationships, and lineage commitment, as well as into the molecular pathways governing these processes (for review, see Kondo et al. 2003; BuzaVidas et al. 2007; Iwasaki and Akashi 2007). However, the importance of RNA surveillance, and in particularly NMD, has yet to be analyzed within the hematopoietic system.

To determine the importance of NMD in the context of a mammalian organism, we generated a conditional mouse knockout of $U p f 2$ and used the hematopoietic system as a model to test the functional consequences of disrupting the NMD pathway. In these mice, ablation of UPF2 led to the fast, complete, and lasting extinction of the hematopoietic stem and progenitor compartment. In contrast, differentiated cells were only mildly affected, suggesting a particular important role of NMD in proliferating cells. Deletion of Upf2 within the T-cell compartment was associated with the accumulation of $\mathrm{PTC}^{+}$ Tcrb transcripts in immature T-cells. In striking contrast, $\mathrm{PTC}^{+} \mathrm{Tcrb}$ transcripts did not accumulate in mature Upf2-null T-cells, suggesting that it is the specific accumulation of $\mathrm{PTC}^{+}$-containing Tcrb transcripts and not the general loss of NMD activity that is deleterious for resting T-cells. Finally, at the molecular level, we could show the importance of NMD in suppression of pseudogene expression and as a modulator of alternative splicing (AS).

\section{Results}

To test the role of NMD in the development and maintenance of the hematopoietic system, we targeted the locus encoding one of the essential NMD factors, UPF2. Given that the Upf2 gene spans $>20$ exons in a $>200-\mathrm{kb}$ region on chromosome 2 , we adopted a targeting strategy where, by flanking exons 2 and 3 with $\operatorname{lox} P$ sites, we could inactivate Upf2 in a conditional manner (Fig. 1A). Correct targeting of transfected ES cells was verified by Southern blotting, and following germline transmission, the resulting $U p f 2^{\text {neostop/+ }}$ mice were used to generate the conditional $U p f 2^{f 1}$ allele by in vivo removal of the 
A

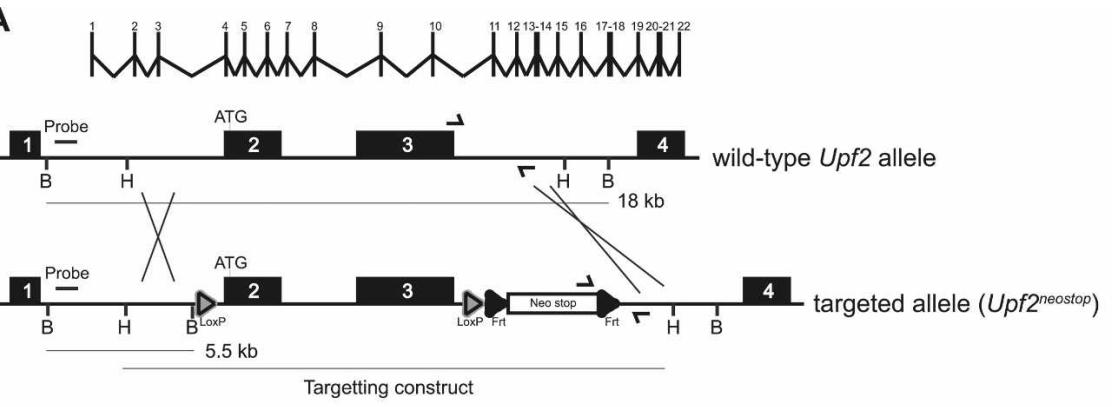

B
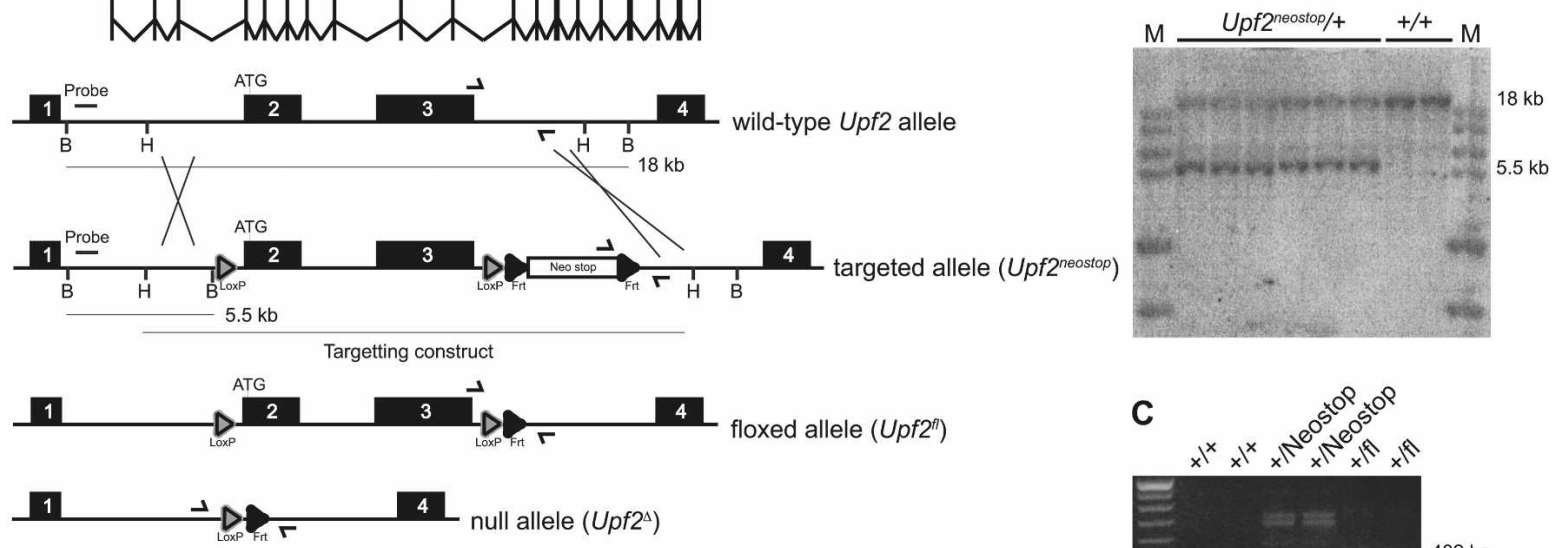

D
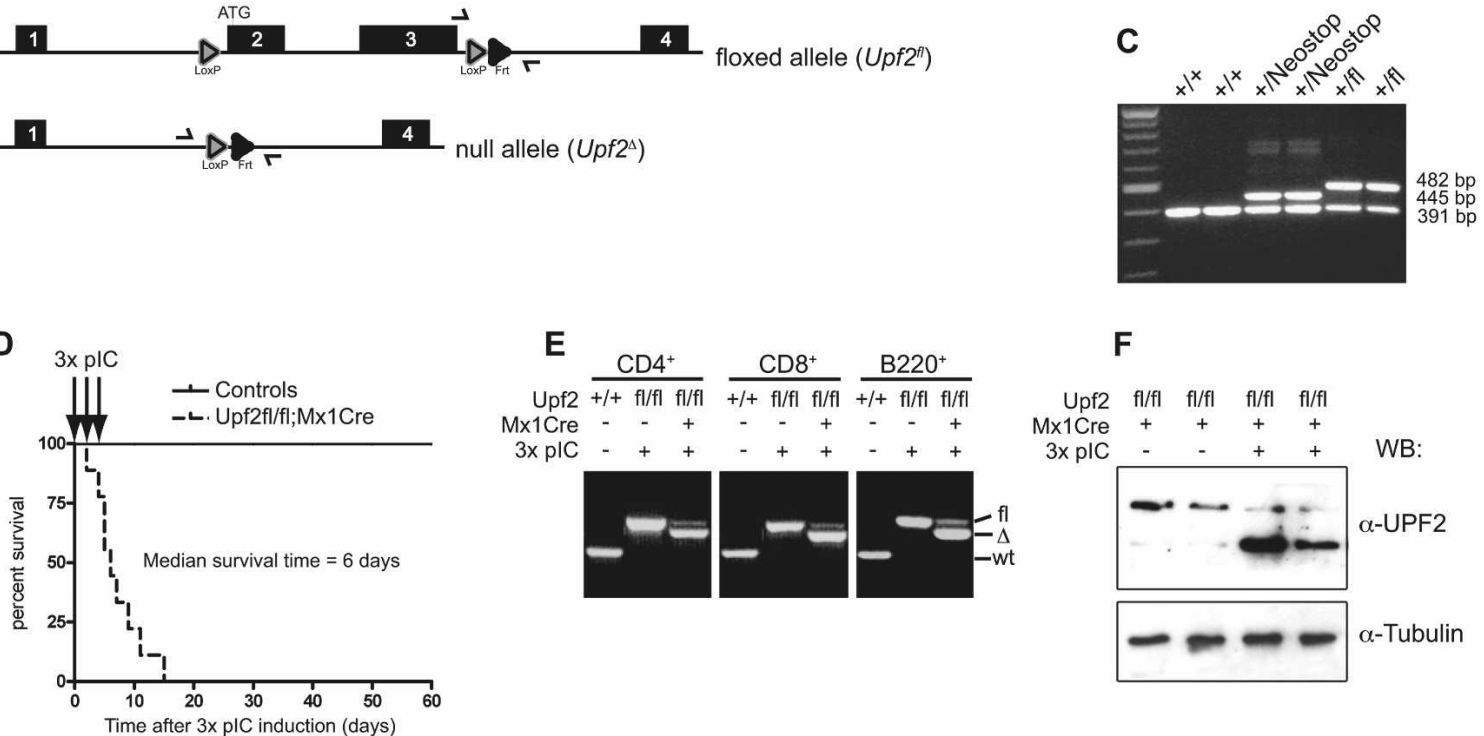

$\mathbf{F}$

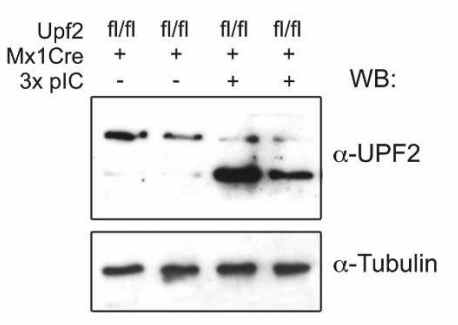

Figure 1. Mx1Cre-mediated conditional deletion of UPF2 leads to rapid mortality. (A) The genomic structure of the $>200-\mathrm{kb} U p f 2$ locus is depicted. The targeting strategy involved flanking exons 2 (containing the initiating ATG) and 3 with loxP sites. Removal of the FRT-flanked Neo cassette by crossing with FLP mice resulted in the generation of the conditional $U p f^{f 1}$ allele. The conditional $U p f 2^{f 1}$ allele is converted to the recombined $U p f 2^{\Delta}$ allele by the Cre recombinase. Primers used for genotyping of the various $U p f 2$ alleles are indicated. $(B)$ Southern blot verification of correctly targeted ES cells. DNA was digested with EcoRI and probed with a probe $5^{\prime}$ to the sequence within the targeting construct. (C) PCR genotyping of $U p f 2^{f l /+}$ crossed to Flp transgenics. $(D)$ pIC injections were performed at days 0,2 , and 4 and led to the rapid death of $U p f 2^{f l / f 1} ; M x 1 C r e(N=9)$, whereas no mortality was observed in any of the different pIC-injected controls (all other genotypes $N=25$ ). (E) PCR genotyping of MACS-purified CD4 ${ }^{+}, \mathrm{CD}^{+}$, and $\mathrm{B}^{2} 20^{+}$splenocytes isolated from pIC-injected $U p f 2^{f l / f l} ; M x 1 C r e$ mice (day 6) and relevant controls. Recombination levels were $>85 \%$. Given the fast clearance of cells upon UPF2 truncation, we were unable to assess recombination levels in the BM. $(F)$ Western blot analysis of lysates from $\mathrm{B} 220^{+} U p f 2^{f l / f 1} ; M \times 1 C r e$ splenocytes (day 6 after the first pIC injection) demonstrates the expression of a truncated UPF2 form in recombined cells.

Frt-flanked Neostop cassette (Fig. 1B,C). Cre-mediated recombination of the $U p f 2^{f l}$ allele resulted in the generation of the $U p f 2^{\Delta}$ allele, which would lead to either ablation of UPF2 protein or to the generation of a truncated form of UPF2 initiating at one of several downstream ATGs. We predicted that such a truncated UPF2 protein would be functionally inactive as it would have deleted several domains of functional importance including the $\mathrm{N}$-terminal part, which is required for UPF1 interaction and NMD (Supplemental Fig. S1A).

To test the effect of Upf2 deletion on embryonic development, we intercrossed $U p f 2^{\Delta /+}$ heterozygotes and isolated embryos at distinct developmental time points. No homozygous $U p f 2^{\Delta / \Delta}$ embryos could be detected at embryonic day 9.5 (E9.5), whereas genotyping of E3.5 blastocysts revealed the expected Mendelian ratio (Supplemental Table S1). These findings show that
UPF2, like UPF1 (Medghalchi et al. 2001), is essential for early embryonic development in the mouse.

\section{Conditional ablation of UPF2 in the hematopoietic system}

In order to probe the role of NMD during hematopoietic development, we crossed the Mx1Cre transgene onto our $U p f 2^{f l /+}$ line. In this model, expression of Cre recombinase can be induced within the entire hematopoietic system upon activation of the Mx1Cre transgene either by administering interferon directly or through a dsRNAinduced interferon response (Kuhn et al. 1995). Ablation of UPF2 within the hematopoietic compartment was accomplished by injecting $U p f 2^{f l / f 1}$;Mx1Cre mice three times with polyinosinic-polycytidylic acid (pIC) at days 0,2 , and 4 . This procedure led to the death of all 
$\mathrm{Upf}^{f 1 / f 1}$;Mx1Cre animals within $10 \mathrm{~d}$ from the first pIC injection (Fig. 1D), whereas no mortality was observed in any of the pIC-injected control animals $\left(U p f 2^{f l / f 1}\right.$, $\left.U p f 2^{+/+}, U p f 2^{+/+} ; M x 1 C r e\right)$. Analysis of the BM from pICinjected $U p f 2^{f 1 / f 1}$;Mx1Cre mice at day 6 (i.e., $2 \mathrm{~d}$ after the last pIC injection) revealed an almost complete absence of nucleated cells (Fig. 2E). Similarly, flow cytometric analysis demonstrated a strong reduction of all major lineages of the BM including hematopoietic progenitors $\left(\mathrm{HPCs}\right.$; c-Kit ${ }^{+}$) (Fig. 2A), granulocytes $\left(\mathrm{Mac}-1^{+} \mathrm{Gr}-1^{+}\right.$) (Fig. 2B) and B-cells $\left(\mathrm{B} 220^{+}\right)$(Fig. 2C). The only cells present in the BM after pIC injection of $U p f 2^{f 1 / f 1}$;Mx1Cre mice were CD71 ${ }^{-}$Ter1 $19^{+}$erythroid cells (Fig. 2D). The finding that the latter cells accumulate in pIC-injected controls at the expense of $\mathrm{CD} 71^{+}$Ter $119^{+}$cells suggests that their accumulation is independent of UPF2 depletion but is a consequence of the induced interferon response (Fig. 2D; Supplemental Fig. S1C). Consistent with the rapid loss of hematopoietic cells in the BM, depletion of UPF2 was accompanied by severe anemia (Fig. 2G). The possibility that interferon (induced by pIC) synergizes with the loss of UPF2 to mediate the strong hematopoietic phenotype is rendered highly unlikely by the opposite effects that pIC injection has on control and $U p f 2^{f 1 / f 1}$;Mx1Cre HPCs (Fig. 2F).

Collectively, these findings demonstrate that UPF2 is essential for the maintenance of the hematopoietic compartment within the BM. In contrast, we could not detect any changes in the numbers of peripheral B- and $\mathrm{T}$-cells in neither spleen nor lymph nodes, suggesting that resting lymphocytes are resistant to acute deletion of UPF2, at least at an early time point (data not shown).

The recombined $U p f 2^{\Delta}$ allele is recessive and encodes a truncated form of UPF2

In order to verify efficient recombination of the $U p f 2^{f 1}$ allele, we isolated $\mathrm{CD}^{+}, \mathrm{CD}^{+}$, and $\mathrm{B}^{2} 20^{+}$splenocytes from pIC-injected $U p f 2^{f 1 / f 1} ; M x 1 C r e$ mice. Allele-specific PCR demonstrated the efficient conversion $(>85 \%)$ to the recombined $U p f 2^{\Delta}$ allele in splenocytes in as little as $6 \mathrm{~d}$ after the first pIC injection (Fig. 1E). At the protein level, we detected a truncated $\sim 120$-kDa form of UPF2 in recombined cells, demonstrating that translation of mRNA derived from the $U p f 2^{\Delta}$ allele initiates at a downstream ATG (Fig. 1F). A protein of similar size was also observed in nonrecombined cells, suggesting that it may be a natural isoform of UPF2 initiating at codon 403 . We next tested whether the $120-\mathrm{kDa}$ UPF2 displayed any dominant-negative or gain-of-function properties. Expression of the $120-\mathrm{kDa}$ UFP2 were found to interfere neither with NMD in transfected HeLa cells nor with hematopoietic homeostasis in pIC-injected $\mathrm{Upf}^{\mathrm{fl/+}}{ }_{;} \mathrm{Mx} 1 \mathrm{Cre}$ mice (Supplemental Fig. S1B,C). These findings demonstrate that it is the loss of full-length UPF2 and not the expression of the $120-\mathrm{kDa}$ isoform that promotes the strong hematopoietic phenotype observed in pICinjected $U p f 2^{f l / f 1} ; M x 1$ Cre mice.
UPF2 is necessary for maintenance of the hematopoietic system in a cell-autonomous manner

The Mx1Cre transgene is also expressed in nonhematopoietic cells, including liver and intestine (Kuhn et al. 1995). Therefore, in order to test the intrinsic effects of deleting Upf2 on hematopoiesis, we took advantage of the CD45.1/CD45.2 system to generate BM chimeras by transplanting $U p f 2^{f l / f 1}$;Mx1Cre BM (CD45.2) into lethally irradiated wild-type recipients (CD45.1) and vice versa (Fig. 3A). [CD45.1 and CD45.2 are alloantigens of CD45. They are used to distinguish cell populations of different origin in transplantation experiments.] pIC injection was initiated $16 \mathrm{wk}$ after transplantation and was found to be fatal for all wild-type recipients transplanted with $U p f 2^{f l / f 1}$;Mx1Cre BM (Fig. 3A). In contrast, only half of the chimeras (two out of four) generated by transplantation of wild-type BM died within our 3-wk observation period and did so by nonhematopoietic phenotypes (Fig. 3A; data not shown). The hematopoietic phenotype of the $U p f 2^{f l / f 1}$;Mx1Cre BM chimeras was indistinguishable from that of $U p f 2^{f l / f 1} ; M \times 1 C r e$ animals. At day 9 after initiation of pIC injection, the few surviving Upf2 $2^{f l / f 1}$;Mx1Cre BM chimeras were highly anemic (Fig. 3B) and had essentially no nucleated cells within their BM, including granulocytes and progenitors (c-Kit $\left.{ }^{+}\right)$ (Supplemental Fig. S2A; data not shown). Interestingly, UPF2 deletion in the Upf2 $2^{f l / f 1}$;Mx1Cre BM chimeras was associated with the rapid and almost complete loss of the HSC-containing LSK $\left(\mathrm{Lin}^{-}, \mathrm{Sca}-{ }^{+}, \mathrm{c}^{-\mathrm{Kit}^{+}}\right.$) compartment (Fig. 3C). At this time point, control $U p f 2^{f l / f 1}$ BM chimeras also displayed reduced numbers of nucleated cells, and the Sca-1 expression on LSK-HSCs was somewhat affected. This was attributed to the transient and mobilizing effect pIC have on HPCs (Fig. 2F), as no differences were present between uninjected and pIC-injected $U p f 2^{f l / f 1} \mathrm{BM}$ chimeras when interrogated at $3 \mathrm{wk}$ postpIC (data not shown). These findings demonstrate that UPF2 is necessary for maintenance of the BM in a cellautonomous manner and that the pronounced lethality observed upon its deletion can be ascribed to its importance in maintaining hematopoietic homeostasis including the essential HSC compartment.

In order to follow the effects of UPF2 deletion over a longer time span and avoid the complications of analyzing the hematopoietic system during the strong interferon response, we generated BM chimeras by transplanting lethally irradiated CD45.1 recipients with a 1:9 ratio of wild-type (CD45.1/2) and Upf2 ${ }^{f l / f 1}$;Mx1Cre (CD45.2) $\mathrm{BM}$ cells. In this setting, the presence of a small wildtype BM fraction ensures the survival of the BM chimeras and also provides a competitive approach to evaluate the consequences of UPF2 ablation. Following engraftment, these mice were treated with three consecutive pIC injections, and the contributions from donor and recipient populations were followed in the peripheral blood (PB). In contrast to the control cohort, transplanted under identical conditions using $U p f 2^{f l / f 1}$ (CD45.2) test cells, ablation of full-length UFP2 led to a rapid decline in overall donor derived CD45.2 cells in PB (Fig. 3D). 
Analysis of peripheral B-cells and granulocytes $12 \mathrm{wk}$ after UPF2 ablation revealed a fivefold and 25 -fold reduction, respectively, of the $U p f 2^{f l / f l} ; M x 1 C r e$ cell contribu-
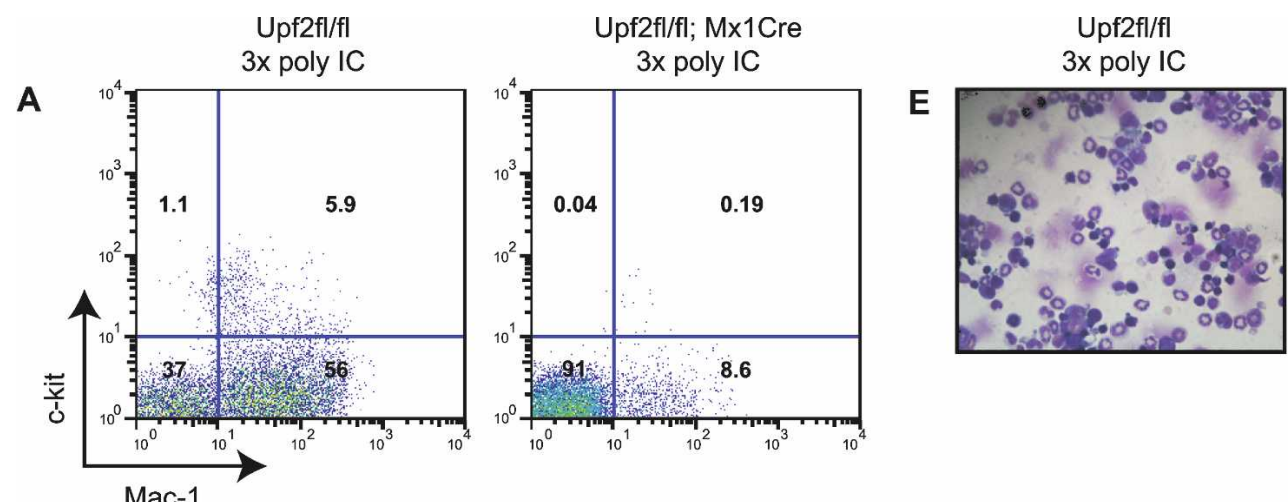

tion to these two lineages (Fig. 3E). As granulocytes need to be continuously supplied from the BM, in contrast to the longer-living B-cells, these findings demonstrate that
B
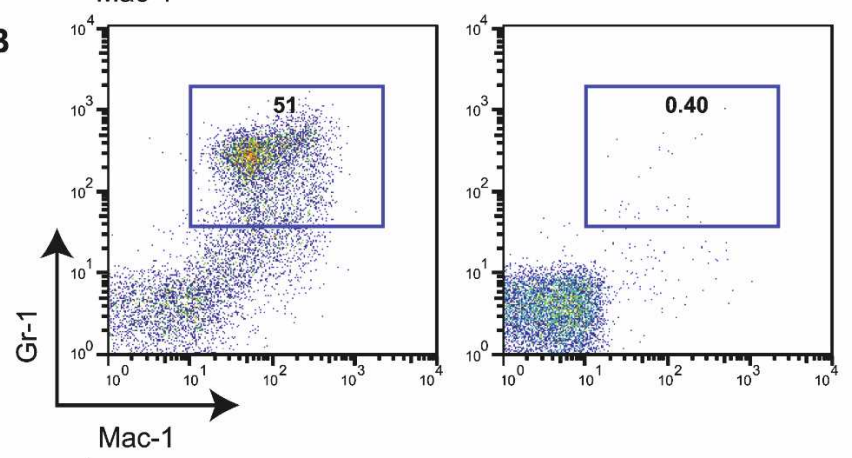

C
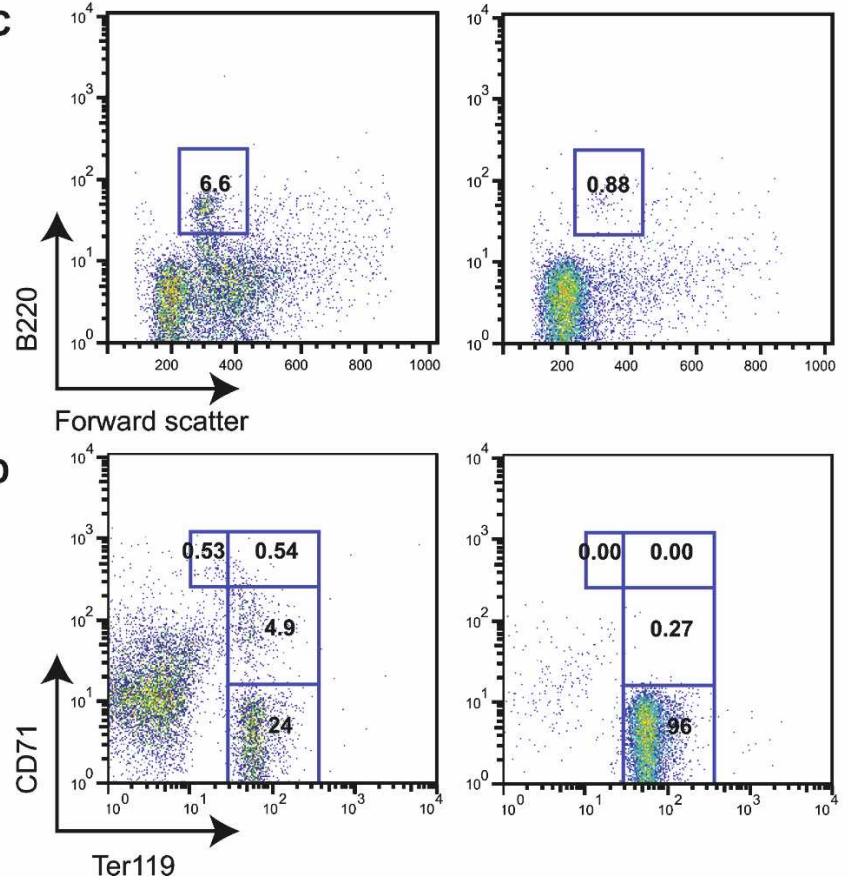

$\mathbf{F}$

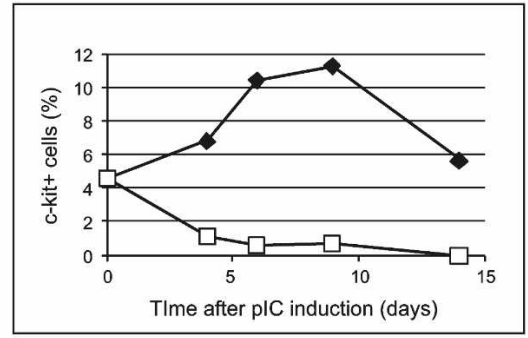

G
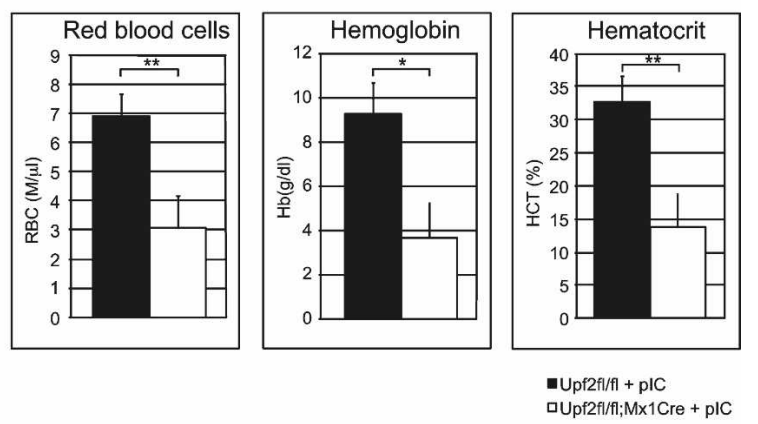

Figure 2. Deletion of full-length UPF2 leads to BM breakdown. $(A-D)$ FACS analysis of BM cells from pIC-injected $U p f 2^{f l / f 1}$ and $U p f 2^{f l / f 1}$;Mx1Cre mice (analyzed at day 6 after initiation of pIC injections). BM cells were stained with directly conjugated antibodies against progenitors $\left(\mathrm{c}-\mathrm{Kit}^{+}\right)$, granulocytes $\left(\mathrm{Macl}^{+} \mathrm{Grl}^{+}\right)$, B-cells $\left(\mathrm{B} 220^{+}\right)$, and erythroid cells $\left(\mathrm{CD} 71^{+/-}\right.$Ter1 $\left.19^{+/-}\right)$. Immature erythroid cells are $\mathrm{CD} 71^{+}$Ter $119^{+/-}$and gradually lose their $\mathrm{CD} 71^{+}$expression during maturation. (E) Cytospins of the same BM cells as described above. $(F)$ Quantification of c-Kit ${ }^{+}$HPC progenitors (as assayed by FACS) in the BM of control Upf $2^{f l / f 1}$ (black diamonds) and $U p f 2^{f 1 /}$ f1; $M x 1 C r e$ mice (white squares) following pIC injection. $(G)$ Erythroid parameters in the PB of the pIC-injected $U p f 2^{f 1 / f 1}$ (black bars) and $U p f 2^{f l / f 1} ; M x 1 C r e$ mice (day 6 after the first pIC injection). Parameters were measured using a Hemavet $950 \mathrm{FS} .\left(^{\star}\right) P<0.05 ;\left(^{\star \star}\right) P<0.01$. 
Weischenfeldt et al.

A

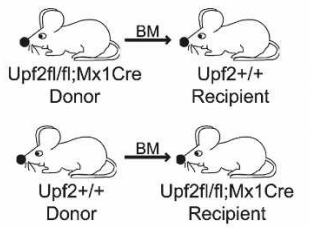

C No support cells; Day 9

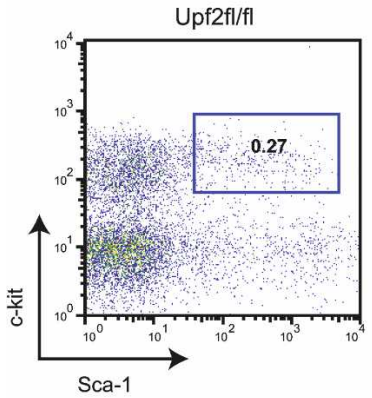

D

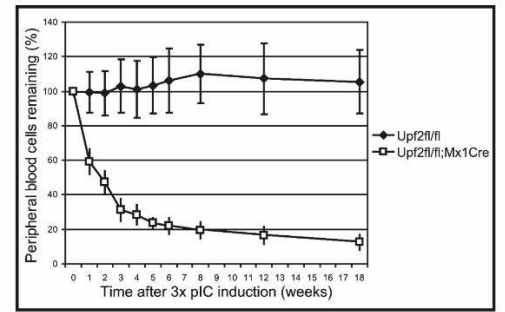

E

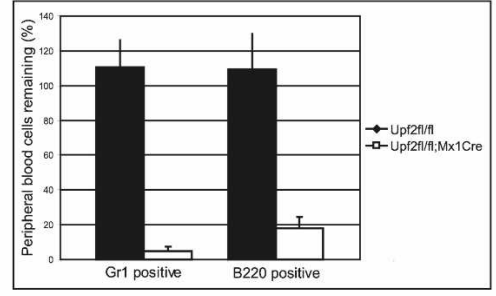

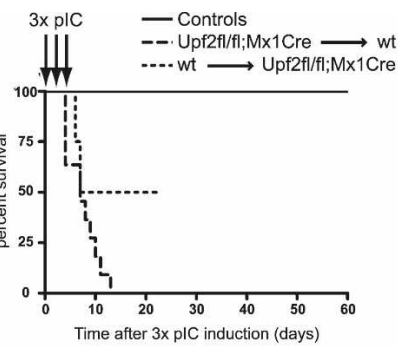

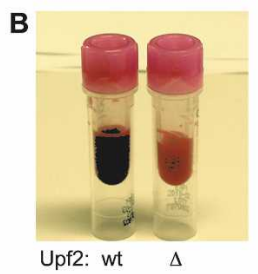

Upf2: wt $\Delta$
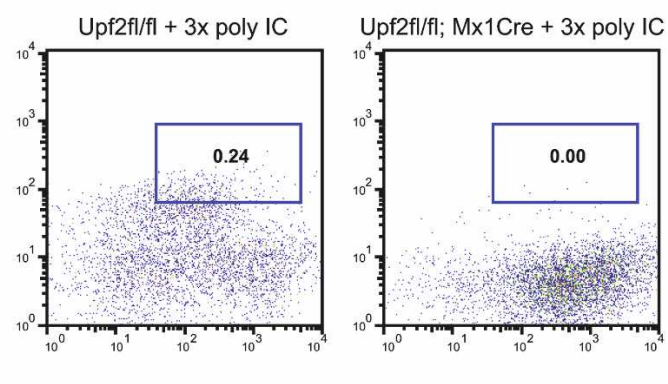

$10 \%$ CD45. 1 support cells; Week 16

F

Upf2fl/fl $+3 x$ poly IC

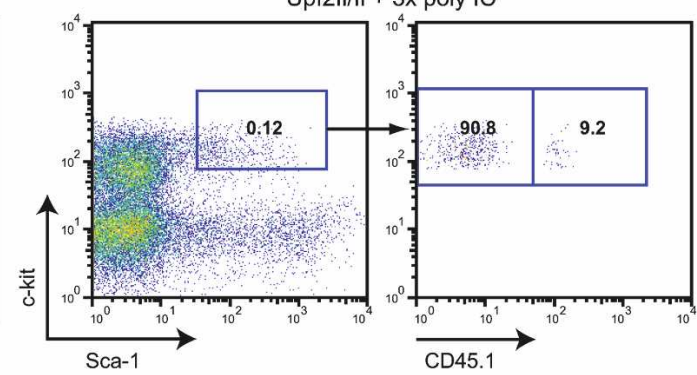

Upf2fl/fl; Mx1Cre + 3x poly IC

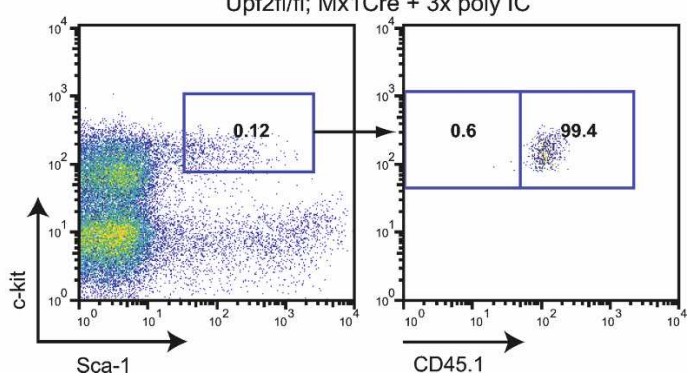

Figure 3. The rapid mortality upon UPF2 deletion is a cell-autonomous feature of BM cells. $(A)$ Lethally irradiated recipient mice were transplanted with 2 million cells as indicated and left for $16 \mathrm{wk}$ before UPF2 deletion. pIC injection led to rapid mortality of all mice transplanted with $U p f 2^{f l / f 1}$;Mx1Cre BM $(N=11)$. (B) PB from control and $U p f 2^{f 1 / f 1}$;Mx1Cre transplanted animals at day 9 after pIC injection demonstrating severe anemia upon UPF2 deletion. (C) FACS analysis of $\mathrm{Lin}^{-}, 7 \mathrm{AAD}^{-}$subsets from BM chimeras showing the rapid loss of both the c-Kit ${ }^{+}$HPC and the HSC-containing LSK populations upon UPF2 deletion (day 9 post-pIC). Please note that the interferon response leads to up-regulation of Sca-1 expression in pIC-injected control mice. LSK frequencies are related to total BM. (D) CD45.2 contribution to PB of recipient mice transplanted with a 9:1 mixture of $U p f 2^{f l / f 1}$;Mx1Cre (or control) (CD45.2) and support cells (CD45.1/2). After $5 \mathrm{wk}$ of engraftment, mice were subjected to pIC injections, leading to the selective loss of CD45.2 ${ }^{+}$ $U p f 2^{f 1 / f 1} ; M x 1 C r e$ cells. Data are presented as the mean \pm SD of a cohort of 10 UPF2 ablated and eight control mice. $(E)$ Lineage analysis of PB isolated from the BM chimeras from $D$ at week 12 post-pIC. Data are presented as the mean \pm SD of 10 UPF2 ablated and eight control mice. (F) FACS analysis of $\mathrm{Lin}^{-}, 7 \mathrm{AAD}^{-}$subsets from $\mathrm{BM}$ chimeras from $D$ analyzed at week 16 post-pIC. UPF2 deletion leads to a selective loss of CD45.2 $\mathrm{Upf}^{\mathrm{fl} / \mathrm{fl}} ; \mathrm{Mx} 1 \mathrm{Cre}$ (depicted as CD45.1-).

UPF2 deletion leads to long-term ablation of hematopoiesis. Analysis of the BM compartment at the experimental endpoint (18 wk after pIC injection) demonstrated that whereas $U p f 2^{f l / f 1}$ cells contributed massively to all tested lineages including B-cells, granulocytes, progenitors (Supplemental Fig. S2B-D), and LSK cells (Fig. 3F), 
the contribution of $U p f 2^{f 1 / f 1} ; \mathrm{Mx} 1 \mathrm{Cre}\left(\mathrm{CD} 45.1^{-}\right)$was reduced to background levels. These data clearly demonstrate a long-term cell-autonomous requirement of UPF2 for hematopoietic maintenance.

To rule out the possibility that the absence of LSK cells could be due to their exhaustion during the course of the experiment, we analyzed additional BM chimeras transplanted with a similar 1:9 ratio of wild-type (CD45.1) and $U p f 2^{f l / f 1} ; M x 1 C r e(C D 45.2)$ BM cells. Sixteen weeks post-transplantation, the animals were injected with three consecutive pIC injections and analyzed at day 18 , which is the earliest time point possible if we are to avoid the pIC effects in control mice (data not shown). Consistent with our previous analysis, UPF2 deletion led to the striking and complete replacement of CD45.2 HSCs (LSKFlt3-) and c-Kit ${ }^{+}$progenitors cells with CD45.1 control cells (Fig. 4A). These findings demonstrate an obligate requirement of UPF2 for the maintenance of the HSC and c-Kit ${ }^{+}$progenitor compartments.

\section{Loss of full-length UPF2 leads to rapid loss of HPCs} in vivo and HPC activity in vitro

The rapid loss of c-Kit ${ }^{+}$cells upon deletion of full-length UPF2 in the BM could be caused by a specific effect on these cells or by down-regulation of the c-Kit receptor itself. We therefore assayed the levels of progenitor populations with low or absent c-Kit expression. Consistent with a general effect on all tested stem and progenitor populations, UPF2 ablation led to a pronounced downregulation in both common lymphoid progenitors (CLP, as assayed by CD45.2 donor contribution) and B-cell progenitors (Fig. 4B; Supplemental Fig. S3).

We next tested the in vitro performance of myeloid progenitors and LT-HSCs. First, we used a colony-forming assay to demonstrate that UPF2-deleted BM cells were devoid of any myeloid colony-forming potential (Fig. 4C). Second, prospectively isolated HSCs (LSKFlt3-) from control and $U p f^{f l / f 1} ; M \times 1 C r e$ mice were subjected to a 3-d in vitro treatment with interferon (to facilitate UPF2 deletion) during and after which the growth of the cultures was tested. Deletion of UPF2 resulted in a robust cessation of growth and in the appearance of highly dysmorphic Upf2 ${ }^{\Delta / \Delta}$ cells (Fig. 4D; Supplemental Fig. S4).

Collectively our phenotypic analysis of the BM deletion of UPF2 demonstrates a unique and absolute requirement for UPF2, and by inference NMD, for the maintenance of all tested stem and progenitor subsets within the BM.

Loss of full-length UPF2 impact on T-cell development, clearance of PTC $C^{+} T c r b m R N A$, and in vitro stimulation of lymphocytes

Having demonstrated the importance of full-length UPF2 for the maintenance of hematopoiesis within the $\mathrm{BM}$, we next sought to address the phenotypical consequences of deleting UPF2 during development of the
T-cell lineage. To that end we used the T-cell-specific deleter LckCre, in which expression of the Cre recombinase has been reported to be activated at the doublenegative stage (DN; $\left.\mathrm{CD}^{-} \mathrm{CD} 8^{-}\right)$during thymic T-cell development (Hennet et al. 1995). In this system, deletion of UPF2 (Fig. 5A) resulted in thymic atrophy with significant reductions in the total numbers of both double-positive (DP; $\mathrm{CD}^{+} \mathrm{CD}^{+}$[threefold]) and singlepositive (SP; $\mathrm{CD}^{+}$[fourfold to fivefold] and $\mathrm{CD}^{+}$[twofold to threefold]) T-lymphocytes (Fig. 5B; Supplemental Fig. S5A). Furthermore, the reduction of $U p f^{f 1 / f 1} ;$ LckCre thymocytes was accompanied by increased numbers of Annexin $\mathrm{V}^{+}$preapoptotic cells (Fig. 5C).

Programmed DNA rearrangements at the Tcr loci occur in immature $\mathrm{CD} 4^{-} \mathrm{CD} 8^{-} \mathrm{DN}$ T-cells and are essential for generating the large functional repertoire of the immune system. Only one in three recombination events is expected to lead to transcripts with full coding potential, and the remaining $\mathrm{PTC}^{+}$Tcrb transcripts (encoding the TCR- $\beta$ protein) are most likely cleared from thymocytes by NMD (Carter et al. 1995; Li and Wilkinson 1998). To test this directly, RNA isolated from either SP T-cells or whole thymus derived from $U p f 2^{f l / f 1}$;LckCre and control mice was subjected to a RT-PCR protocol across the V-D-J recombination junctions of the Tcrb transcript (Supplemental Fig. S6). The Tcrb PCR products were cloned, and the resultant inserts were subjected to sequencing. Whereas no $\mathrm{PTC}^{+}$fragments were identified in any sequences derived from wild-type mice, $29 \%$ and $27 \%$ of the sequences derived from SP and total $U p f^{f l / f 1} ;$ LckCre thymocytes, respectively, contained PTCs as a result of nonproductive rearrangements (Fig. $5 \mathrm{D})$. These findings unequivocally demonstrate that the NMD pathway is highly active in T-cells and that its abrogation leads to accumulation of $\mathrm{PTC}^{+} \mathrm{Tcrb}$ transcripts.

We consistently observed $\mathrm{CD}^{+}$and $\mathrm{CD} 8^{+} \mathrm{SP} \mathrm{T}$-cells in spleen and lymph nodes from $U p f 2^{f 1 / f 1}$; LckCre mice at $\sim 30 \%$ of the wild-type control levels (Supplemental Fig. S5A,B). In contrast to what was found in thymocytes, sequencing of $T c r b$ cDNA did not reveal any appreciable accumulation of $\mathrm{PTC}^{+} \mathrm{Tcrb}$ transcripts in Upf2-null peripheral T-cells (one out of 26), suggesting that an aberrantly rearranged $T c r b$ allele is deleterious to resting T-cells (Fig. 5D). We then asked whether the peripheral T-cells without PTC $^{+}$Tcrb mRNAs had indeed lost the expression of full-length UPF2. Surprisingly, we could detect fully recombined peripheral T-cells (Supplemental Fig. $5 \mathrm{C}, \mathrm{D})$, as measured by recombination of the Upf2 ${ }^{\Delta}$ allele in spleen and lymph node from Upf2 $2^{f / \Delta \text { neostop; }}$ LckCre mice (the Upf2 ${ }^{\Delta \text { neostop }}$ allele is another null allele derived by recombination of the original Upf2 $2^{\text {neostop }}$ allele). This implies that whereas resting peripheral $\mathrm{T}$-cells are unable to tolerate $\mathrm{PTC}^{+} \mathrm{Tcrb}$ transcripts, they can survive in the absence of UPF2 and, by inference, NMD.

Having demonstrated that UPF2 is required for proper T-cell development but appears dispensable in peripheral T-cells lacking $\mathrm{PTC}^{+}$Tcrb transcripts, we next asked whether acute disruption of UPF2 in mature T-cells had any impact on their ability to be activated in response to exogenous stimuli. We therefore isolated splenocytes 
Weischenfeldt et al.

A $\quad 10 \%$ CD45.1 support cells; Day 18

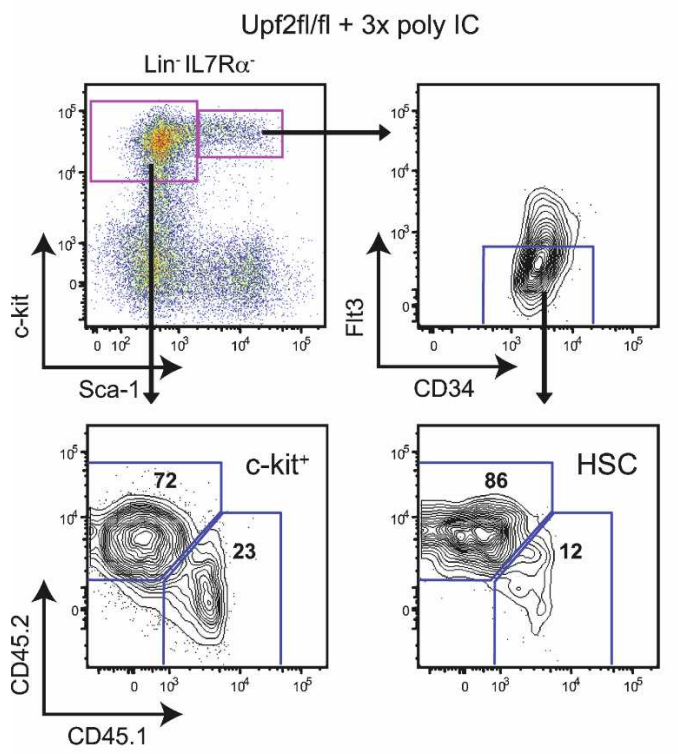

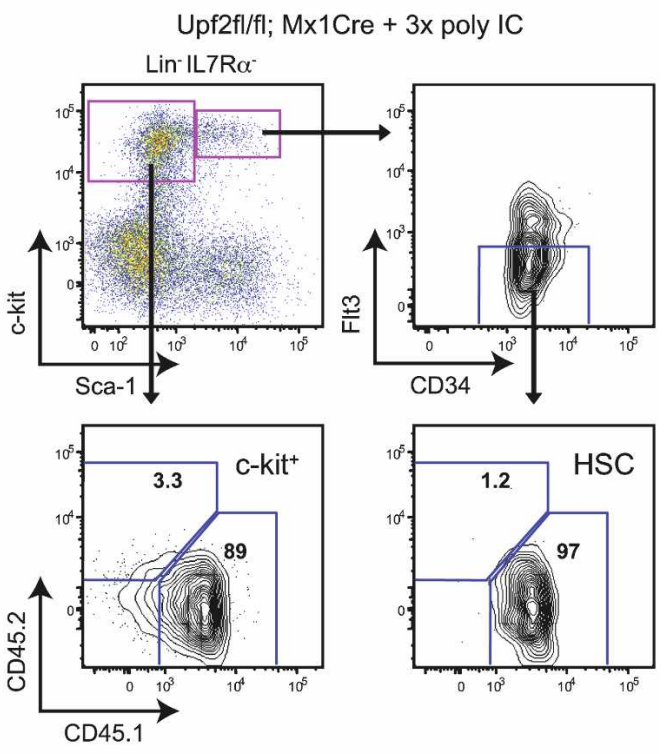

Upf2fl/fl; Mx1Cre $+3 x$ poly IC

Lin-IL7R $\alpha$

Upf2fl/fl; Mx1Cre $+3 x$ poly IC

Upf2fl/fl $+3 x$ poly IC

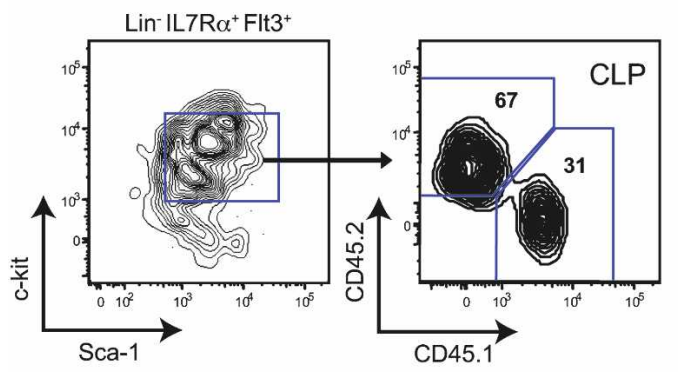

$7 \mathrm{R}^{+} \mathrm{FIt}^{+}$

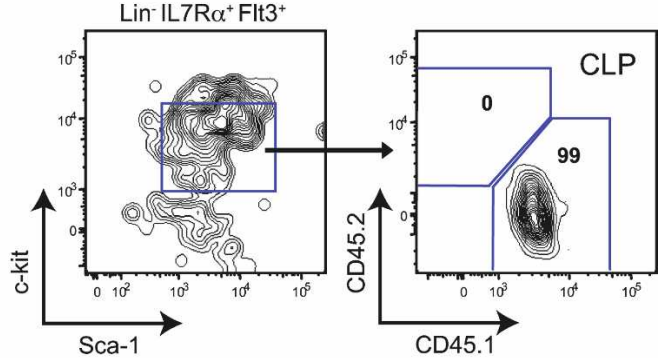

C

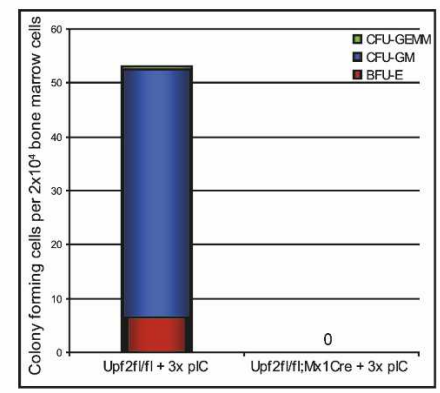

D

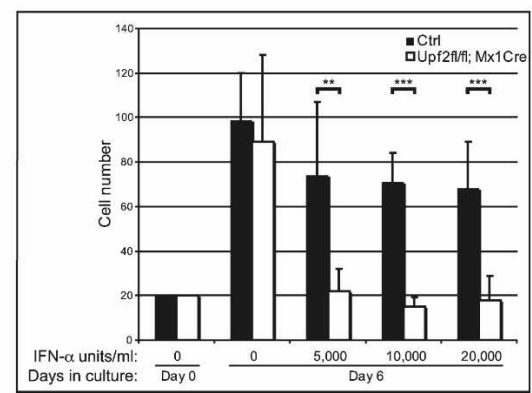

Figure 4. UPF2 deletion leads to fast clearance of the entire HSC and progenitor compartment. (A) FACS analysis of Lin ${ }^{-}$, IL-7R $\alpha^{-}$BM subset from lethally irradiated recipients transplanted with a 9:1 mixture of $U p f 2^{f l / f 1} ; M x 1 C r e$ or control (CD45.2) and support cells (CD45.1). Sixteen-week post-transplant mice were subjected to $3 \times$ pIC injections and analyzed at day 18 . UPF2 deletion leads to loss of both c-Kit ${ }^{+}$progenitors and HSCs (LSKFlt3 ${ }^{-}$). (B) FACS analysis of Lin-IL-7R $\alpha^{+} \mathrm{Flt}^{-}{ }^{-}$BM subset from the mice from $A$ demonstrating the strong selection against $U p f 2^{\Delta / \Delta}$ cells within the CLP fraction. $(C)$ The number of colony-forming units (CFUs) of BM cells derived from pIC-injected ( $3 \times$; cells plated at day 6) $U p f 2^{f 1 / f 1}$ and $U p f 2^{f 1 / f 1} ; M x 1 C r e$ mice. Colonies were scored after $12 \mathrm{~d}$ in $\mathrm{M} 3434 \mathrm{medium}$ as either multipotent CFU-GEMM (granulocyte/erythrocyte/macrophage/megakaryocyte), committed myeloid CFU-GM (granulocyte/ macrophage), or committed erythroid BFU-E (burst-forming unit; erythrocyte). More than 200,000 Upf2 ${ }^{f 1 / f 1}$;Mx1Cre BM cells were assayed. $(D)$ FACS-sorted LSKFlt3- cells were grown for $3 \mathrm{~d}$ in 96-well plates, in the absence or presence of IFN- $\alpha \mathrm{A} / \mathrm{D}$, in medium containing thrombopoietin and stem cell factor. Growth was continued for another $3 \mathrm{~d}$ after which the numbers of cells were scored. Each data point is averaged from six individual wells (means of triplicates of sorted cells from two different mice of each genotype $\left.\pm \mathrm{SD}) .\left(^{\star \star}\right) P<0.01{ }^{* \star \star \star}\right) P<0.001$. 
from pIC-treated $U p f 2^{f 1 / f 1} ; M x 1 C r e$ animals, labeled them with CFSE, and stimulated their proliferation in vitro. Strikingly, deletion of UPF2 led to a profound reduction in the ability of both $\mathrm{CD}^{+}{ }^{+}$and $\mathrm{CD} 8^{+} \mathrm{SP}$ T-cells to enter the cell cycle in response to exogenous stimuli (Fig. 5E). Similar observations were made when mature B-cells were stimulated in vitro, demonstrating the common requirement of resting lymphocytes for functional UPF2 for activation-induced cell cycle entry.

Collectively, these data demonstrate that functional UPF2, and by inference NMD, is required for the development of T-cells, for the clearance of nonfunctional Tcrb transcripts, and for the ability of lymphoid cells to re-enter the cell cycle in response to extracellular stimuli. Finally, the increased accumulation of proapoptotic cells in the thymus strongly suggests that UPF2depleted thymocytes are cleared by apoptosis.

\section{Deletion of UPF2 is tolerated in the myeloid lineage}

Given the strong phenotypic consequences of UPF2 deletion in both the HSC/HPC compartments of the BM, it could not be established whether the observed reductions in cells of the myeloid lineages were secondary to impaired stem and progenitor function or due to UPF2 being directly required for the integrity of myeloid cells. Thus, we next addressed the functional consequences of specifically deleting UPF2 in the myeloid lineage. To that end we crossed the $U p f 2^{f 1}$ allele into the LysMCre knock-in strain, which has been reported to express Cre recombinase mainly in the granulocytic/monocytic lineage (Clausen et al. 1999). Surprisingly, FACS analysis of BM cells isolated from Upf2 ${ }^{f l / f 1}$;LysMCre animals did not reveal any reduction in the numbers of either granulocytes or monocytes, despite the significant reduction of full-length UPF2 in the former (Fig. 6A,B).

We next tested the impact of UPF2 deletion during Macrophage Colony-Stimulating Factor (M-CSF)-mediated differentiation of BM cells. This in vitro differentiation protocol resulted in essentially pure populations of BM-derived macrophages (BMMs), which were able to phagocytose irrespectively of their UPF2 status (Fig. $6 \mathrm{~B}, \mathrm{C})$. Transfection with wild-type and $\mathrm{PTC}^{+} \beta$-globin reporter constructs demonstrated that $U p f 2^{f 1 / f 1}$; LysMCre BMMs were indeed devoid of any NMD activity (Fig.
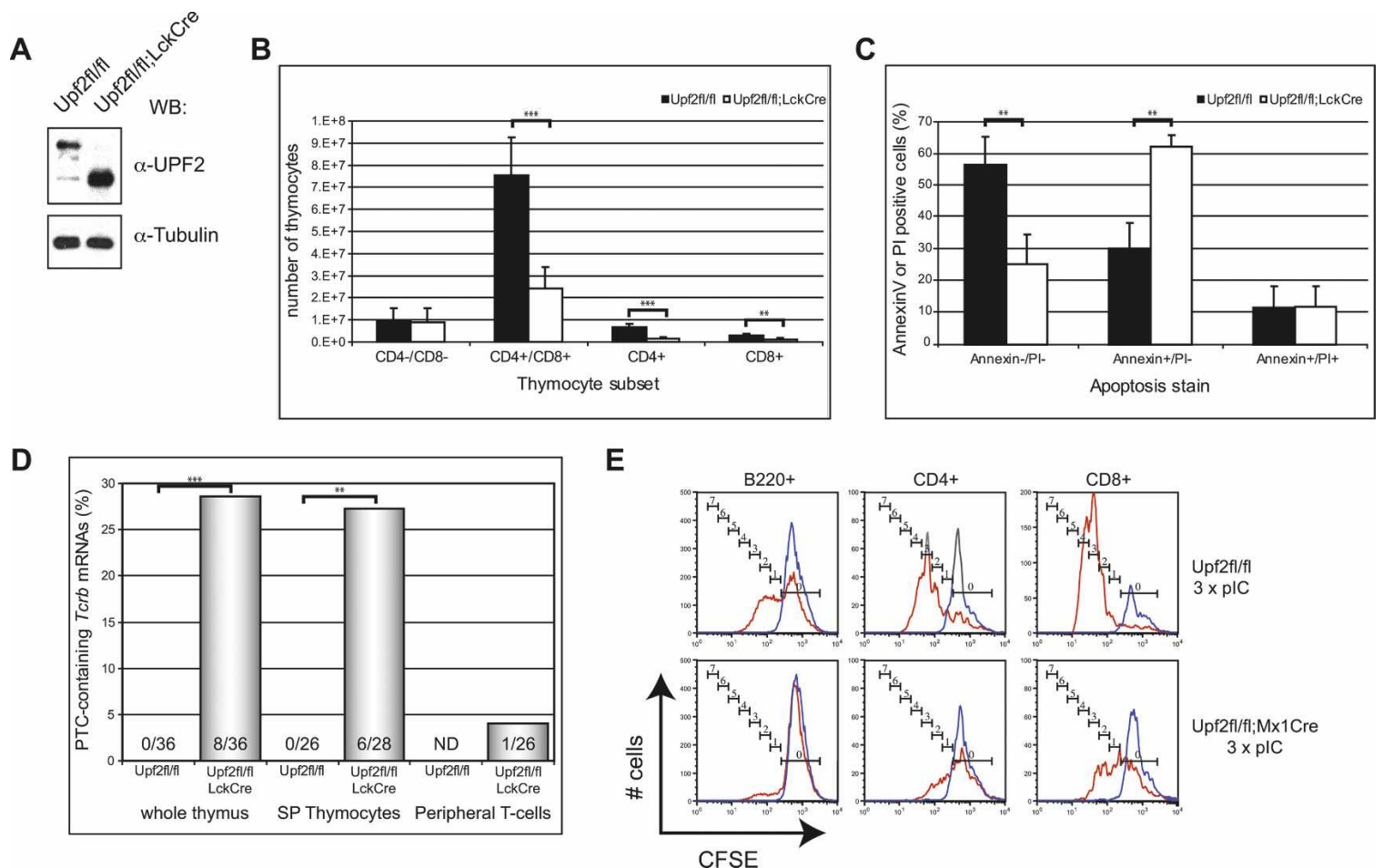

Figure 5. T-cell-specific loss of UPF2. (A) Western blot showing efficient recombination of UPF2 in thymus $\left(U p f 2^{f 1 / f 1} ;\right.$ LckCre). $(B)$ Quantification of thymocyte subsets from $U p f^{f 1 / f 1}$; LckCre mice, based on the expression of CD4 and CD8. (C) UPF2 deletion leads to the accumulation of early apoptotic thymocytes in $U p f 2^{f l / f 1}$; LckCre mice as assayed by the presence of $A n n e x i n V^{+}, \mathrm{PI}^{-}$cells. $(D)$ Accumulation of $\mathrm{PTC}^{+}$Tcrb mRNAs in UPF2-deficient thymocytes but not mature peripheral cells $\left(U p f 2^{f 1 / f 1} ;\right.$ LckCre $)$ as assayed by a strategy involving RT-PCR, cloning, and sequencing (Supplemental Fig. S6). Numbers of PTC ${ }^{+}$sequences $(x)$ and numbers of total clones sequenced $(y)$ are given for each condition $(x / y)$. (E) CFSE-labeled UPF2-deficient splenocytes $\left(U p f 2^{f l / f l} ; M x 1 C r e\right)$ failed to proliferate in response to mitogenic stimuli. Splenocytes (day 6; i.e., $2 \mathrm{~d}$ after the last pIC injection) were stimulated in vitro for $72 \mathrm{~h}$ with either LPS (B-cells) or CD3 + CD28 antibodies (T-cells); stained with conjugated B220, CD4, or CD8 antibodies; and assayed by FACS analysis. Blue and red lines indicate nonstimulated and stimulated cells, respectively. The numbers of cell divisions, as given by the "Proliferation" feature of FlowJo, are shown. Data in $B$ and $C$ are presented as the mean \pm SD of four UPF2 ablated and 11 control mice. $\left(^{\star \star}\right) P<0.01 ;\left(^{\star \star \star}\right) P<0.001$. 
Weischenfeldt et al.

6D). Finally, when BM cells were pulse-labeled with CFSE at either day 2 or day 4 during a 7 -d M-CSF-mediated in vitro differentiation protocol, we did not observe any proliferative disadvantage in $U p f 2^{f 1 / f 1}$;LysMCre BMMs (Fig. 6E).

Collectively, these findings demonstrate that UPF2, and by inference NMD, is dispensable during terminal differentiation of the myeloid lineage.

Gene expression profiling of $U p f 2^{\Delta / \Delta} B M M s$ and thymocytes

Knockdown experiments in transformed human cells suggested that NMD components had extensive effects on the regulation of $4 \%-10 \%$ of the transcriptome (Mendell et al. 2004; Wittmann et al. 2006). To examine the importance of NMD on global transcription under physiological conditions, we subjected BMMs and thymocytes derived from $U p f 2^{f l / f 1}$;LysMCre and $U p f 2^{f l / f 1}$;LckCre mice, respectively, to microarray-based gene expression profiling (Supplemental Tables S2, S3). Both these cell types show complete ablation of NMD as assayed by transfection with reporter constructs or sequencing of Tcrb transcripts (Figs. 5D, 6D). Upf $2^{\Delta / \Delta}$ BMMs displayed significant changes (greater than twofold; $P<0.05$ ) in the expression of 189 genes (234 probe sets) of which 186 were up-regulated. Similarly, in $U p f 2^{\Delta / \Delta}$ thymocytes, we found the expression of 37 genes ( 42 probe sets) to be significantly changed with only two of these being down-regulated (Fig. 7A). Close to $75 \%$ (26 out of 35 ) of the up-regulated genes in thymocytes were also up-regulated in BMMs, thereby identifying these as core UPF2 targets (Fig. 7C). Most of these transcripts have features of bona fide NMD targets including upstream ORFs (uORFs) and 3' untranslated region (UTR) introns or can generate $\mathrm{PTC}^{+}$isoforms through AS (Fig. 7B,C). The

Figure 6. UPF2 deletion is well-tolerated within the myeloid lineage. (A) FACS analysis of BM from control and $U p f 2^{f 1 / f 1}$. ;LysMCre mice demonstrates no alterations in the frequencies of either Mac- $1^{+} \mathrm{Gr}-1^{+}$granulocytes or $\mathrm{Macl}^{+} \mathrm{F} 4 / 80^{+}$monocytes. (B) Western blot analysis of MACS-purified Gr- $1^{+} \mathrm{BM}$ cells and in vitro derived BMMs demonstrate a partial (albeit significant) and complete deletion of UPF2, respectively. $(C)$ The phagocytic index of BMMs, measured by the average number of ingested Zymosan A particles per 100 BMMs, reveals no functional alterations upon deletion of UPF2. Data are presented as the mean \pm SD of three mice per genotype. $(D)$ The ability of UPF2deleted BMMs to perform NMD is totally abolished as assayed by transient transfections of BMMs with NMD-sensitive (pc $\beta 39)$ and NMD-insensitive $(\mathrm{pc} \beta \mathrm{WT})$ reporter constructs. Band intensities on the Northern blot were normalized to a control (pc $\beta G)$, and relative resistance toward NMD is shown as the ratio between $\mathrm{pc} \beta 39$ and $\mathrm{pc} \beta \mathrm{WT}$ for a given genotype (see Supplemental Fig. S1 for details). (E) FACS-based CFSE analysis of BMM outgrowth labeled at either day 2 or day 4 during a 7 -d differentiation protocol. No significant changes in the loss of CFSE labeling during BMM differentiation was observed between $U p f 2^{f 1 / f 1}$ (blue lines) and $U p f 2^{f 1 / f 1}$;ysMCre (red lines).

strong bias toward up-regulation of transcript levels is consistent with the removal of a mRNA degradation component.

Within the conserved UPF2 core set, we noticed three well-described SnoRNA host genes-Snord22, Snhg6, and Gas5 (Fig. 7C,D). SnoRNAs belong to the ever-expanding world of small noncoding RNAs and are mainly described as serving as site-specific guides for post-transcriptional modifications of rRNA, but also for other RNAs (Kiss et al. 2006). In addition, six of the eight up-
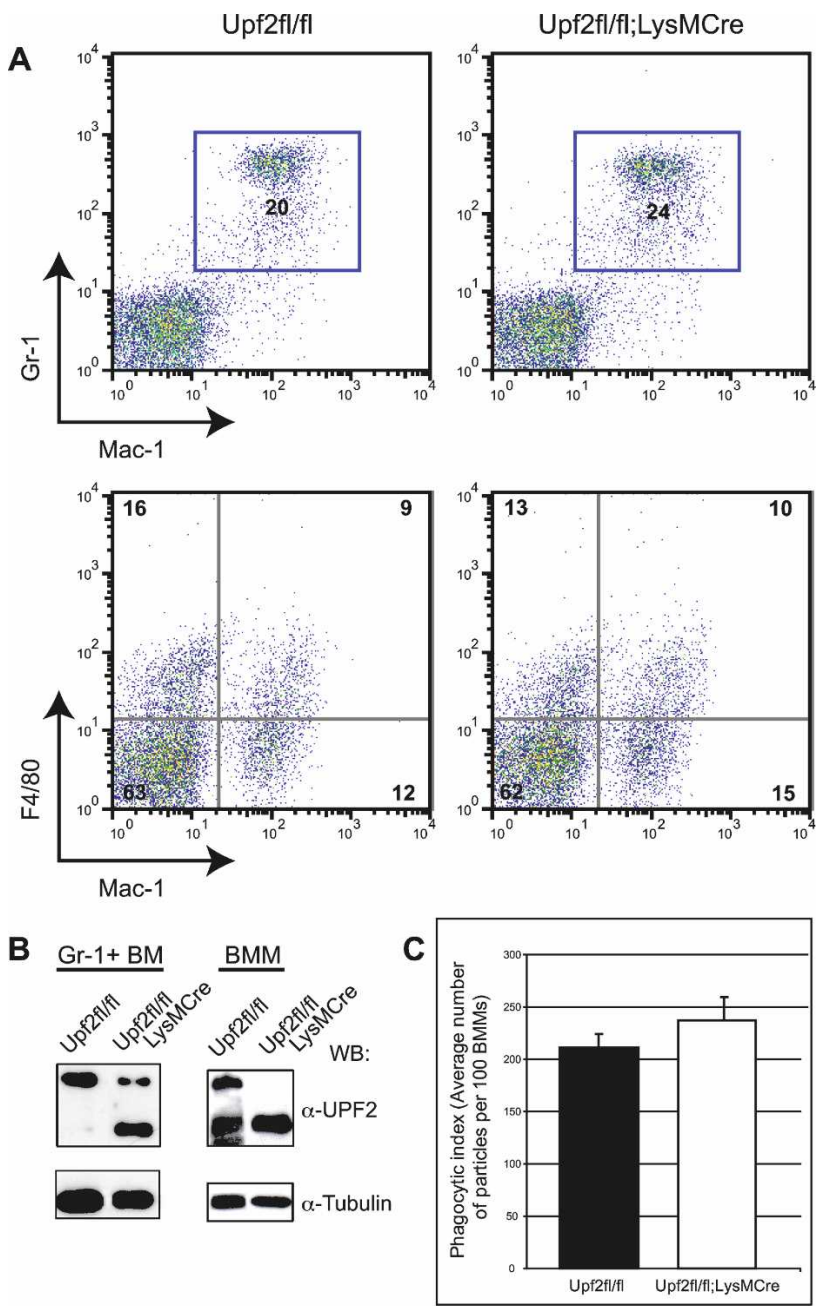

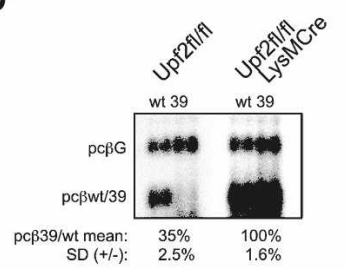

E

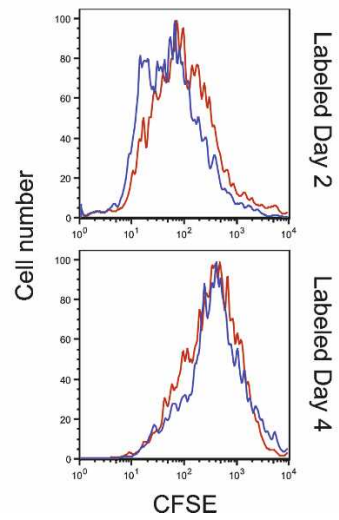




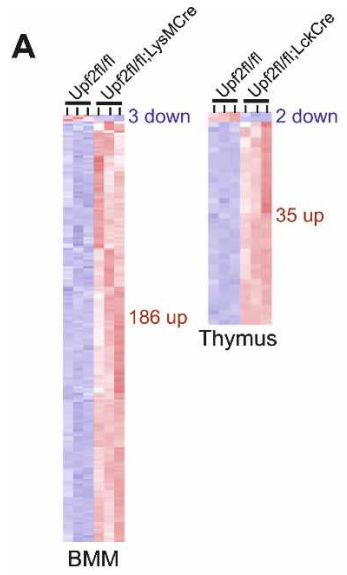

B Putative NMD features

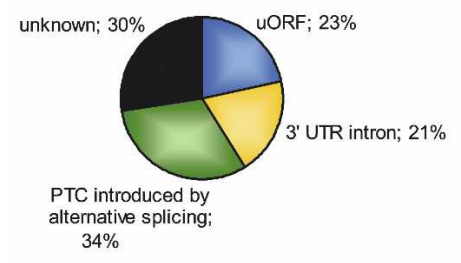

D

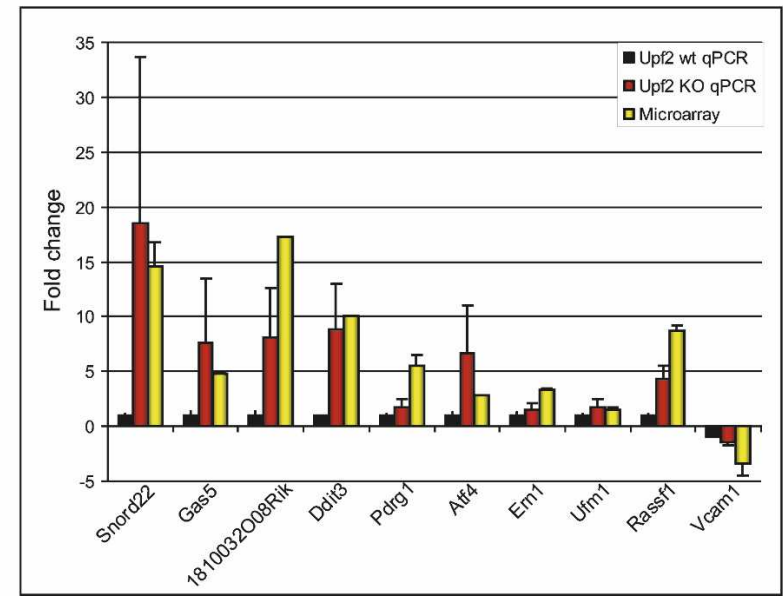

UPF2 Core Set

\begin{tabular}{|lccclll|}
\hline & & \multicolumn{3}{l|}{ Fold Change } & & \\
\cline { 3 - 5 } Gene name & Entrez gi & BMM & Thymus & NMD feature & Gene Ontology \\
2310005L22Rik & 69471 & 20.45 & 7.58 & $3^{\prime}$ UTR intron & snoRNA host gene \\
1810032008Rik & 66293 & 17.25 & 4.94 & AS & snoRNA host gene \\
Snord22 & 83673 & 16.6 & 4.6 & $3^{\prime}$ UTR intron & snoRNA host gene \\
Pvt1 & 19296 & 14.84 & 2.95 & AS & Unknown \\
2810026P18Rik & 72655 & 11.69 & 5.78 & $3^{\prime}$ UTR intron & snoRNA host gene \\
Ddit3 & 13198 & 10.04 & 2.8 & uORF & Unfolded protein response \\
1500012F01Rik & 68949 & 9.97 & 4.92 & AS & snoRNA host gene \\
OTTMUSG00000010657 & 666532 & 8.34 & 2.67 & unknown & Regulation of transcription \\
Polg2 & 50776 & 7.24 & 2.47 & AS & DNA repair \\
Bbs4 & 102774 & 7.2 & 2.7 & unknown & microtubule cytoskeleton organization \\
Sirt3 & 64384 & 6.93 & 3.34 & unknown & Chromatin silencing \\
Gas5 & 14455 & 4.9 & 2.56 & $3^{\prime}$ UTR intron & snoRNA host gene \\
Mrpl49 & 18120 & 4.82 & 2.35 & unknown & Translation initiation \\
Pdrg1 & 68559 & 4.75 & 2.39 & AS & Unfolded protein binding \\
Tatdn1 & 69694 & 4.33 & 2.97 & AS & DNase activity \\
Snhg6 & 73824 & 4.16 & 3.25 & $3^{\prime}$ UTR intron & snoRNA host gene \\
D10Ertd641e & 52717 & 3.77 & 2.64 & unknown & Unknown \\
Trub2 & 227682 & 3.76 & 2.49 & $3^{\prime}$ UTR intron & RNA processing \\
2410006H16Rik & 69221 & 3.75 & 5.53 & uORF & snoRNA host gene \\
5530601H04Rik & 71445 & 3.74 & 2.63 & unknown & Unknown \\
2010109N14Rik & 69895 & 3.49 & 2.64 & uORF & snoRNA host gene \\
2310073E15Rik & 75692 & 3.48 & 2.59 & unknown & Putative receptor \\
Nud13 & 67725 & 2.9 & 2.81 & Unknown & Putative hydrolase activity \\
Atf4 & 11911 & 2.77 & 2.54 & uORF & Transcriptional regulation, Unfolded protein response \\
Arf2 & 11841 & 2.7 & 2.55 & unknown & Protein transport \\
Urm1 & 68205 & 2.37 & 2.92 & unknown & Protein degradation \\
\hline & & & & &
\end{tabular}

E

PTC exon-inclusion targets in BMMs
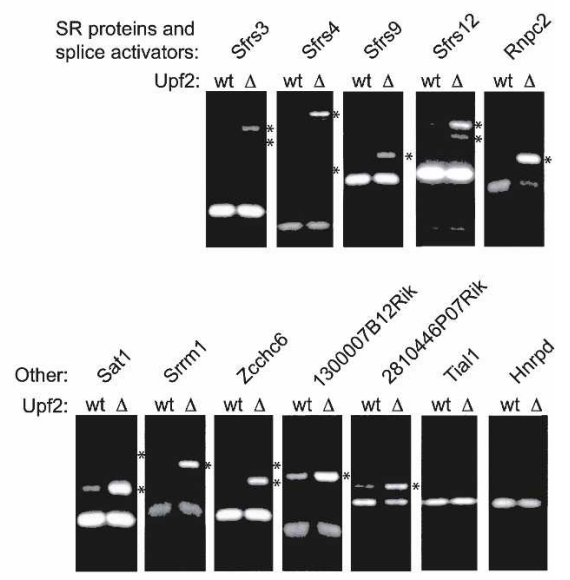

Figure 7. Deletion of UPF2 derepresses snoRNA host genes and interferes with the autoregulatory circuitry regulating the levels of splicing activators. (A) Microarray-based expression analysis of UPF2-depleted BMMs and thymocytes (a detailed list is given in Supplemental Tables S2, S3). Note the strong bias toward up-regulation. The numbers of deregulated genes (greater than twofold; $P<0.05)$ are given to the right of the heat maps. The data were obtained from three independent samples of each genotype (males, pairwise litter-matched). (B) Graphical representation of NMD features for the 60 most up-regulated genes in the BMM data set. (C) The UPF2 core set constitutes genes that are deregulated upon UPF2 deletion in both BMMs and thymocytes. (D) Quantitative RT-PCR validation of a subset of genes in the UPF2 core set. The fold change of the deregulation for individual genes are depicted as determined by quantitative PCR (red) and microarray (yellow). Data are presented as the mean \pm SD of three mice per genotype. (E) Deletion of UPF2 affects the distribution of alternative splice isoforms of mRNAs containing highly or ultraconserved elements (Ni et al. 2007). RT-PCR analysis of RNA isolated from BMMs shows that the PTC ${ }^{+}$AS transcripts were stabilized for 10 out of 12 tested genes (including five known splicing activators). Splice isoforms that are up-regulated in the absence of UPF2 are indicated by an asterisk.

regulated Riken sequences in our core set were also found to contain SnoRNAs within their introns upon manual inspection. Thus, $35 \%$ of the genes in the UPF2 core set serve as SnoRNA host genes, representing a total of 23 different SnoRNA genes (Supplemental Table 4).
These findings demonstrate an unexpected requirement for NMD in down-regulation of several SnoRNA host genes.

The UPF2 core set also contained several genes (Ddit3, Atf4, Ufm1, Ern1, Pdrg1) involved in endoplasmic re- 
ticulum (ER) stress, protein degradation, and the unfolded protein response (UPR). The latter pathway senses unfolded proteins in the ER by various sensors and initiates a program resulting in an increase in ER folding capacity, temporary cessation of protein synthesis, and, if the unfolded protein load is too severe, apoptosis (Ron and Walter 2007). The up-regulation of UPR genes in UPF2-depleted cells could be due to global up-regulation of $\mathrm{PTC}^{+}$transcripts encoding truncated proteins with reduced folding potential leading to the induction of UPR. However, we were unable to detect a significant difference in the UPR of UPF2-depleted BMMs and thymocytes, as assayed by up-regulation of the ER chaperone $\mathrm{BiP}$, increased phosphorylation of the general translation initiation factor eIF $2 \alpha$, or increased cleavage of Xbp1 premRNA (Supplemental Fig. S7), suggesting that at least in these cells, the UPR pathway is not activated in response to loss of NMD. Possibly the presence of NMD-sensitive UPR transcripts (e.g., Atf4) could reflect the existence of an autoregulatory loop, where UPR-mediated inhibition of protein synthesis (and thereby NMD) leads to the selective and sustained stabilization of key UPR transcripts during UPR.

\section{Ablation of NMD results in stabilization of alternatively spliced transcripts containing PTC}

AS has been hypothesized as a mechanism to extend the protein-coding diversity without expanding the number of genes. Approximately one-third of all AS transcripts contain a PTC, and NMD has been proposed to safeguard the cell from these potentially deleterious transcripts (Lewis et al. 2003). Previous reports using knockdown of UPF1 have demonstrated that a fraction of $\mathrm{PTC}^{+} \mathrm{AS}$ transcripts is destabilized by NMD, a mechanism coined AS coupled with NMD (AS-NMD) (Pan et al. 2006; Ni et al. 2007). Interestingly, a group of transcripts with highly conserved $\mathrm{PTC}^{+}$exons were enriched among AS-NMD targets, and splicing activators (e.g., SR proteins) and splicing repressors (e.g., hnRNP proteins) were further overrepresented in this group, suggesting that their levels could be regulated by AS-NMD (Lareau et al. 2007; Ni et al. 2007). To test whether these findings were valid in primary cells, we performed RT-PCR on alternatively spliced mRNAs previously identified as $\mathrm{PTC}^{+}$exon targets (Ni et al. 2007), to determine if the levels of the AS $\mathrm{PTC}^{+}$transcripts were stabilized in $U p f 2^{\Delta / \Delta}$ BMMs (Fig. $7 \mathrm{E})$. For 10 out of 12 genes tested we could indeed show that the AS $\mathrm{PTC}^{+}$isoform was up-regulated in NMD ablated cells. Significantly, all five tested splice activators were verified. These findings suggest that NMD plays an important role in vivo in modulating regulated AS events.

\section{Discussion}

The NMD pathway has emerged as an important posttranscriptional mechanism that eliminates $\mathrm{PTC}^{+}$ mRNAs, thereby prohibiting the synthesis of potential harmful protein products. Although having been extensively studied at the biochemical level, this study represents the first in-depth analysis of the NMD pathway in the context of a mammalian organism.

Since UPF2, in contrast to several of the other NMD components, has not been associated with non-NMD functions, we chose to target the Upf2 locus. Similar to the previously characterized Upf1-null mice, mice homozygous for our functional Upf2-null allele died during early embryogenesis, further substantiating NMD as an essential pathway during fetal development.

We sought to model the importance of NMD in one of the central adult self-renewing organ systems, the BM. Here, induced deletion of UPF2 led to fast removal of all nucleated cells, including $\mathrm{c}^{-\mathrm{Kit}^{+}}$progenitor cells, in a cell-autonomous manner, and death of the animals within 5-10 d. We demonstrated that LSK cells are completely depleted from BM in as little as $9 \mathrm{~d}$ after initiating UPF2 deletion. More elaborate experiments using mixed $\mathrm{BM}$ chimeras (to ensure survival of the transplanted animals) confirmed this finding and allowed us to demonstrate that UPF2 loss is associated with a fast, complete, and lasting extinction of the HSC compartment.

In sharp contrast to the profound effect of UPF2 deletion in hematopoietic stem and progenitor cells, we observed essentially no phenotypic consequences of deleting UPF2 in the myeloid lineage. Moreover, peripheral Band T-cells were only mildly affected upon acute UPF2 deletion despite the Upf2 locus being efficiently recombined. These observations are suggestive of a specific function of UPF2 in proliferating cells, a hypothesis consistent with the inability of peripheral $U p f 2^{\Delta / \Delta}$ B- and $\mathrm{T}$-cells to enter the cell cycle upon mitogenic stimuli. With respect to the rarely dividing HSCs, we hypothesize that the fast clearance of progenitor cells upon UPF2 deletion either forces HSCs into cycle, leading to their rapid exhaustion, or that these cells become unable to coordinate proliferation/differentiation events and are cleared by apoptosis. Thus our data on the phenotypic description of the hematopoietic system are consistent with an obligate requirement for UPF2, and by inference NMD, for the survival of proliferating, but not terminally differentiated, cells.

NMD has been reported to be of particular importance in lymphoid cells (Li and Wilkinson 1998). During their maturation, B- and T-cells undergo extensive programmed DNA rearrangements involving the genes encoding Ig and TCR, respectively. Although these rearrangement events, which are necessary in order to generate the diverse immune repertoire, are predicted to result in a significant fraction of nonproductively rearranged alleles of these genes, $\mathrm{PTC}^{+}$Tcrb transcripts are not accumulating in wild-type T-cells. In contrast, $\sim 28 \%$ of $T c r b$ transcripts from NMD-deficient $U p f 2^{\Delta / \Delta}$ thymocytes contain PTCs. Strikingly, PTC $^{+}$Tcrb transcripts did not accumulate to any appreciable extent in mature T-cell populations despite the presence of Upf2-null, and therefore, NMD ablated T-cells. These observations suggest two interrelated conclusions: First, resting T-cells appear to display a unique requirement for NMD-medi- 
ated down-regulation of $\mathrm{PTC}^{+}$Tcrb transcripts, as opposed to a general requirement for NMD activity. Secondly, in the absence of NMD, resting T-cells expressing a nonproductively rearranged $T c r b$ allele are selected against during T-cell maturation. Thus, the NMD pathway appears to improve the efficiency of T-cell maturation by facilitating the survival of cells carrying both a nonproductively and a productively rearranged Tcrb allele, and we speculate that this could be generalized to other rearranged lymphoid genes. The reason(s) for the apparent toxicity of a $\mathrm{PTC}^{+} \mathrm{Tcrb}$ transcript in resting T-cells is currently not known; however, one possibility could be that truncated TCR- $\beta$ interferes with the formation of the normal TCR/CD3 complex.

\section{NMD and gene expression}

In contrast to the pronounced effects of deleting UPF2 in stem, progenitor, and T-cells, we observed no gross cellular phenotype when UPF2 was deleted in the myeloid lineage using the LysMCre deleter strain. This allowed us to use an in vitro differentiation protocol to generate $U p f 2^{\Delta / \Delta}$ BMMs, which appeared functionally normal. Comparison of gene expression profiles of wild-type and $U p f 2^{\Delta / \Delta}$ cells revealed a marked preference for up-regulation of transcripts in both thymus $(95 \%)$ and BMMs (98\%) upon ablation of NMD. This observation compares well to expression profiles obtained by knockdown experiments in Drosophila Schneider cells but contrasts to those from human cells, where comparable levels of up- and down-regulated genes were observed (Mendell et al. 2004; Rehwinkel et al. 2005; Wittmann et al. 2006). We hypothesize that this reflects a fundamental difference between heavily mutated mammalian cancer cell lines and primary cells.

Comparison of the gene sets derived from thymus and BMMs yielded a list of 26 core transcripts, which were commonly up-regulated upon deletion of UPF2 in the two cell types. Nine of these represented SnoRNA host genes that encode SnoRNA genes within their introns. Although some, like Gas5, may encode a protein, the exons of these genes are considered to facilitate the presence of the SnoRNA-containing introns and to mediate their appropriate expression. To our knowledge, NMD has not previously been implicated as a regulator of SnoRNA host gene expression in mammalian cells. In $C$. elegans, NMD was reported to down-regulate the expression of several pseudogenes, one of them hosting a single SnoRNA (Mitrovich and Anderson 2005). Here it was proposed that expressed pseudogenes constitute a pool of genes under constant evolution. To allow this extensive probing of the sequence space and to avoid their untimely resurrection, these genes are silenced by NMD, as indeed was shown in C. elegans. Such a mechanism may be particularly relevant in mammalian cells given the current estimates of pseudogenes $(20,000)$ within the human genome (Torrents et al. 2003). Due to their poor representation on standard expression microarrays, our data do not reveal any information as to the global importance of NMD in down-regulation of expressed pseu- dogenes or other ncRNAs. However, we do speculate that the NMD-regulated SnoRNA host genes we identified in the expression profiling analysis of $U p f 2^{\Delta / \Delta}$ cells may represent the tip of an iceberg made up of a large number of expressed pseudogenes. Experiments are underway to probe this possibility. Finally, it should be noted that in addition to the NMD target genes reported here, there could also be mRNAs that are regulated through an UPF2-independent NMD pathway (Gehring et al. 2005).

AS has emerged as a major mechanism of regulating gene expression and most protein-encoding genes have one or more AS transcripts (Blencowe 2006). Analysis of EST sequences revealed that $\sim 10 \%$ are derived from $\mathrm{PTC}^{+}$AS transcripts (Xing and Lee 2004). It would therefore seem reasonable to argue that ablation of NMD would strongly enhance the levels of AS-NMD transcripts with associated fatal consequences (Lewis et al. 2003). However, controversy exists concerning the magnitude of AS-NMD targets (Pan et al. 2006; Ni et al. 2007).

NMD has been convincingly demonstrated to act in a negative feedback loop regulating the activity of $\sim 20$ splicing regulators (Lareau et al. 2007; Ni et al. 2007). Here transcripts containing ultraconserved elements were shown to be involved in regulated AS events in a manner dependent on the inclusion and exclusion of $\mathrm{PTC}^{+}$exons in the transcripts of splicing activators and repressors, respectively. According to this model, an autoregulatory circuit is set up, in which elevated amounts of splicing activators will result in increased levels of an NMD-sensitive transcript (by exon inclusion) leading to reduction of the activator, as exemplified by SFRS3 and SFRS10 (Jumaa and Nielsen 1997; Stoilov et al. 2004), whereas elevated amounts of a splicing repressor will repress the inclusion of a coding exon, again leading to NMD and down-regulation of the repressor (Ni et al. 2007). We tested the NMD dependence on the stabilization of $\mathrm{PTC}^{+}$AS transcripts for a subset of the transcripts regulated by ultraconserved elements in $U p f 2^{\Delta / \Delta} \mathrm{BMM}$ cells. Indeed, we were able to show that even in vivo 10 out of 12 of the tested $\mathrm{PTC}^{+}$AS transcripts were deregulated in an NMD-dependent manner, including SFRS3 and SFRS10. These findings strongly suggest that disruption of the NMD pathway leads to disruption of the homeostatic control of splicing regulators, which in turn may have profound consequences on a plethora of other regulated splicing events.

\section{Conclusions and future directions}

Although we focused on the NMD-related issues in our phenotypic description of the $U p f 2^{\Delta / \Delta}$ mice, there is mounting evidence that some NMD components do have non-NMD functions in mammalian cells (Azzalin and Lingner 2006a,b). Recently it was demonstrated that NMD components, including UPF2 (although to a lesser extent), negatively regulate the accumulation of the telomere-encoded Terra RNA at telomeric regions (Azzalin et al. 2007). Interestingly, alleviation of this repression, 
led to various chromosomal abnormalities, suggesting that NMD components may play a role in telomeric maintenance as previously reported for SMG-6 (Reichenbach et al. 2003). To what extent this is due to an indirect effect of ablating canonical NMD or truly novel functions for these proteins is currently uncertain. Future studies will address to what extent UPF2 deletion may affect telomere functions in vivo.

In summary, we provide compelling evidence for an essential role of UPF2 and, by inference, NMD in the survival and maintenance of hematopoietic stem and progenitor cells. Moreover, we show that in vivo, NMD impacts on several layers of gene regulation, including programmed DNA rearrangements at the Tcrb locus, processing of non-protein-coding genes and regulation of AS. Similar to the global effects observed by disruption of miRNA biogenesis in vivo (Bernstein et al. 2003), our challenge for the future will be to determine the relative contributions of these regulatory mechanisms to the strong phenotype of the $U p f 2^{\Delta / \Delta}$ mice, and to determine to what extent they can be ascribed to the deregulation of individual molecular players, to the global impact conferred by the loss of NMD or to any non-NMD functions of UPF2.

\section{Materials and methods}

Colony assays

For the analysis of colony-forming potential, 20,000 BM cells were plated in semisolid methylcellulose-based medium (M3434; StemCell Technologies) supplied with erythropoietin, IL-3, IL-6, and stem cell factor. After $12 \mathrm{~d}$ in cultures, colonies were scored as CFU-GM, BFU-E, or CFU-GEMM.

\section{$B M$ transplantations}

Recipient mice were lethally irradiated by a single radiation dose (900 cGy) and transplanted with 2 million donor BM cells. For the experiments testing the impact of UPF2 truncation solely in hematopoietic cells, we generated BM chimeras by transplanting B6-SJL (CD45.1) recipients with either $U p f 2^{f 1 / f 1}$ or Upf $2^{f l / f 1}$;Mx1Cre (both CD45.2) BM cells. Donor contributions in the $\mathrm{PB}$ were $>90 \%$. Sixteen weeks after transplantation, mice were subjected to pIC injections.

In order to test the effects of UPF2 truncation in nonhematopoietic cells, we generated reverse BM chimeras by transplanting $U p f 2^{f 1 / f 1}$ or $U p f 2^{f 1 / f 1} ; M \times 1 C r e(C D 45.2)$ recipients with wild-type B6-SJL (CD45.1) BM. Donor contributions in the PB were $>90 \%$. Sixteen weeks after transplantation, mice were subjected to pIC injections.

For the experiments testing the performance of $U p f 2^{f 1 / f 1} ; M \times 1 C r e$ donor cells over time, lethally irradiated B6-SJL (CD45.1) recipients were transplanted with 9:1 mixtures of donor cells (CD45.2 $U p 2^{f 1 / f 1} ; M x 1 C r e$ or $\left.U p f 2^{f 1 / f 1}\right)$ and carrier cells $(\mathrm{CD} 45.1 / 2$ or CD45.1 wild-type cells). Donor contributions in the PB were $>90 \%$. Five weeks or 16 wk after transplantation, mice were subjected to pIC injections.

All mouse work was approved by the Danish Animal Ethical Committee ("Dyreforsøgstilsynet").

Mitogenic stimulation of lymphoid cells

Spleen cells were harvested and red blood cells were lysed in 1 $\mathrm{mL}$ of $\mathrm{RBC}$ lysis buffer $\left(155 \mathrm{mM} \mathrm{NH}_{4} \mathrm{Cl}, 10 \mathrm{mM} \mathrm{KHCO}_{3}, 1 \mathrm{mM}\right.$
EDTA) for $2 \mathrm{~min}$ at room temperature, followed by washing in Lymphocyte medium (RPMI1640 +10\% FCS) supplemented with $20 \mathrm{mM}$ HEPES. Splenocytes $\left(50 \times 10^{6} / \mathrm{mL}\right)$ were resuspended in Lymphocyte medium and CarboxyFluoroscein Succinimidyl Ester (CFSE; CellTrace, Invitrogen) was added to a final concentration of $5 \mu \mathrm{M}$, incubated for $5 \mathrm{~min}$ at room temperature, and washed three times with Lymphocyte media supplemented with $20 \mathrm{mM}$ HEPES. For lymphocyte stimulation, splenocytes $\left(10^{5}\right.$ splenocytes in $200 \mu \mathrm{L}$ of Lymphocyte Medium) were added to round-bottomed 96-well plates in the presence of either $30 \mu \mathrm{g} / \mathrm{mL}$ LPS (Sigma) or $0.5 \mu \mathrm{g} / \mathrm{mL}$ of antiCD3 + anti-CD28 antibodies (eBioscience) to stimulate B- and $\mathrm{T}$-cells, respectively. Following 72 -h incubation at $37^{\circ} \mathrm{C}$, cells were dissociated in $0.5 \mathrm{mM}$ EDTA/PBS for $5 \mathrm{~min}$ at $37^{\circ} \mathrm{C}$, washed, and resuspended in $\mathrm{PBS} / 5 \%$ FCS prior to FACS analysis.

\section{In vitro differentiation and functional assays of BMMs}

BM cells were plated at $2 \times 10^{6} / \mathrm{mL}$ in BMM media (RPMI1640 + Glutamax-I, 10\% FCS, supplemented with 10\% L929-conditioned media [M-CSF source]) and grown for $7 \mathrm{~d}$. BMM medium was replaced every 2-3 d until analysis. For CFSE labeling, cells were labeled with CFSE either at day 2 or 4 during the in vitro differentiation protocol before flow cytometric analysis.

The phagocytotic properties of BMMs were assayed using Alexa-fluor 594-conjugated Zymosan A (Saccharomyces cerevisiae) bioparticles (Invitrogen) according to the manufacturer's instructions. Briefly, particles were concentrated on BMMs by centrifugation followed by a $1-\mathrm{h}$ incubation at $37^{\circ} \mathrm{C}$. Following extensive washing, BMMs were centrifuged onto microscope slides, fixated, and costained with DAPI and phalloidin-FITC (Sigma).

The ability of in vitro differentiated BMMs to perform NMD was assayed by transiently transfecting BMMs using the Nucleofector Kit (Amaxa). Briefly, BMMs $\left(1 \times 10^{6}\right.$ in $100 \mu \mathrm{L}$ of Nucleofector Solution [Amaxa]) were transfected with $1 \mu \mathrm{g}$ of pc $\beta w t G A P 3$ UAC ( $\beta$-globin internal control) and either $1 \mu \mathrm{g}$ of pc $\beta w t$ (NMD-insensitive) or $1 \mu \mathrm{g}$ of pc $\beta 39$ (NMD-sensitive) $\beta$-globin reporter plasmids (Lykke-Andersen et al. 2000) in a cuvette using the Amaxa Nucleofector. After culturing for $48 \mathrm{~h}$, RNA was isolated and subjected to Northern analysis.

\section{DNA microarray analysis}

RNA isolated from BMMs or whole thymus was labeled according to the one-cycle eukaryotic target-labeling protocol (Affymetrix) and hybridized to Affymetrix Mouse Genome 4302.0 GeneChips. The .CEL files were obtained using the Affymetix GCOS version 1.4 software and analyzed using the statistical software R. Affymetrix and gcrma packages were used to correct for optical noise and nonspecific binding and to summarize multiple probes into a single expression value. Default settings for gcrma modeling were used (background correction, quantiles normalization, PM-only modeling, and median polish summarization). Detection calls were determined by MAS5.0 and required $100 \%$ present calls within a replicate group to be considered expressed. Replicate groups were compared using "compare samples" from dChip (Li and Wong 2001) requiring a significant fold change $>2(P<0.05$; Welch $t$-test $)$. Microarray data were deposited in ArrayExpress (accession no. E-MEXP-1371).

\section{Acknowledgments}

This work was supported by the Lundbeck Fundation, The Danish Medical Research Council, The Danish Natural Science Re- 
search Council, and the Copenhagen Hospital Cooperation, Region $\mathrm{H}$. We have no conflicting interests to declare.

\section{References}

Azzalin, C.M. and Lingner, J. 2006a. The double life of UPF1 in RNA and DNA stability pathways. Cell Cycle 5: 1496-1498.

Azzalin, C.M. and Lingner, J. 2006b. The human RNA surveillance factor UPF1 is required for $S$ phase progression and genome stability. Curr. Biol. 16: 433-439.

Azzalin, C.M., Reichenbach, P., Khoriauli, L., Giulotto, E., and Lingner, J. 2007. Telomeric repeat containing RNA and RNA surveillance factors at mammalian chromosome ends. Science 318: 798-801.

Bernstein, E., Kim, S.Y., Carmell, M.A., Murchison, E.P., Alcorn, H., Li, M.Z., Mills, A.A., Elledge, S.J., Anderson, K.V., and Hannon, G.J. 2003. Dicer is essential for mouse development. Nat. Genet. 35: 215-217.

Blencowe, B.J. 2006. Alternative splicing: New insights from global analyses. Cell 126: 37-47.

Buza-Vidas, N., Luc, S., and Jacobsen, S.E. 2007. Delineation of the earliest lineage commitment steps of haematopoietic stem cells: New developments, controversies and major challenges. Curr. Opin. Hematol. 14: 315-321.

Carter, M.S., Doskow, J., Morris, P., Li, S., Nhim, R.P., Sandstedt, S., and Wilkinson, M.F. 1995. A regulatory mechanism that detects premature nonsense codons in T-cell receptor transcripts in vivo is reversed by protein synthesis inhibitors in vitro. J. Biol. Chem. 270: 28995-29003.

Chan, W.K., Huang, L., Gudikote, J.P., Chang, Y.F., Imam, J.S., MacLean II, J.A., and Wilkinson, M.F. 2007. An alternative branch of the nonsense-mediated decay pathway. EMBO $J$. 26: $1820-1830$.

Chang, Y.F., Imam, J.S., and Wilkinson, M.F. 2007. The nonsense-mediated decay RNA surveillance pathway. Annu. Rev. Biochem. 76: 51-74.

Clausen, B.E., Burkhardt, C., Reith, W., Renkawitz, R., and Forster, I. 1999. Conditional gene targeting in macrophages and granulocytes using LysMcre mice. Transgenic Res. 8: 265277.

Conti, E. and Izaurralde, E. 2005. Nonsense-mediated mRNA decay: Molecular insights and mechanistic variations across species. Curr. Opin. Cell Biol. 17: 316-325.

Czaplinski, K., Ruiz-Echevarria, M.J., Paushkin, S.V., Han, X., Weng, Y., Perlick, H.A., Dietz, H.C., Ter-Avanesyan, M.D., and Peltz, S.W. 1998. The surveillance complex interacts with the translation release factors to enhance termination and degrade aberrant mRNAs. Genes \& Dev. 12: 1665-1677.

Fukuhara, N., Ebert, J., Unterholzner, L., Lindner, D., Izaurralde, E., and Conti, E. 2005. SMG7 is a 14-3-3-like adaptor in the nonsense-mediated mRNA decay pathway. Mol. Cell 17: 537-547.

Gehring, N.H., Kunz, J.B., Neu-Yilik, G., Breit, S., Viegas, M.H., Hentze, M.W., and Kulozik, A.E. 2005. Exon-junction complex components specify distinct routes of nonsense-mediated mRNA decay with differential cofactor requirements. Mol. Cell 20: 65-75.

He, F., Brown, A.H., and Jacobson, A. 1997. Upf1p, Nmd2p, and Upf3p are interacting components of the yeast nonsensemediated mRNA decay pathway. Mol. Cell. Biol. 17: 15801594.

Hennet, T., Hagen, F.K., Tabak, L.A., and Marth, J.D. 1995. T-cell-specific deletion of a polypeptide $\mathrm{N}$-acetylgalactosaminyl-transferase gene by site-directed recombination. Proc. Natl. Acad. Sci. 92: 12070-12074.
Isken, O. and Maquat, L.E. 2007. Quality control of eukaryotic mRNA: Safeguarding cells from abnormal mRNA function. Genes \& Dev. 21: 1833-1856.

Iwasaki, H. and Akashi, K. 2007. Myeloid lineage commitment from the hematopoietic stem cell. Immunity 26: 726-740.

Jumaa, H. and Nielsen, P.J. 1997. The splicing factor SRp20 modifies splicing of its own mRNA and ASF/SF2 antagonizes this regulation. EMBO J. 16: 5077-5085.

Kashima, I., Yamashita, A., Izumi, N., Kataoka, N., Morishita, R., Hoshino, S., Ohno, M., Dreyfuss, G., and Ohno, S. 2006. Binding of a novel SMG-1-Upf1-eRF1-eRF3 complex (SURF) to the exon junction complex triggers Upf1 phosphorylation and nonsense-mediated mRNA decay. Genes \& Dev. 20: 355-367.

Kiss, T., Fayet, E., Jady, B.E., Richard, P., and Weber, M. 2006. Biogenesis and intranuclear trafficking of human box C/D and H/ACA RNPs. Cold Spring Harb. Symp. Quant. Biol. 71: 407-417.

Kondo, M., Wagers, A.J., Manz, M.G., Prohaska, S.S., Scherer, D.C., Beilhack, G.F., Shizuru, J.A., and Weissman, I.L. 2003. Biology of hematopoietic stem cells and progenitors: Implications for clinical application. Annu. Rev. Immunol. 21: 759-806.

Kuhn, R., Schwenk, F., Aguet, M., and Rajewsky, K. 1995. Inducible gene targeting in mice. Science 269: 1427-1429.

Lareau, L.F., Inada, M., Green, R.E., Wengrod, J.C., and Brenner, S.E. 2007. Unproductive splicing of SR genes associated with highly conserved and ultraconserved DNA elements. Nature 446: $926-929$.

Lewis, B.P., Green, R.E., and Brenner, S.E. 2003. Evidence for the widespread coupling of alternative splicing and nonsensemediated mRNA decay in humans. Proc. Natl. Acad. Sci. 100: $189-192$.

Li, S. and Wilkinson, M.F. 1998. Nonsense surveillance in lymphocytes? Immunity 8: 135-141.

Li, C. and Wong, W.H. 2001. Model-based analysis of oligonucleotide arrays: Expression index computation and outlier detection. Proc. Nat1. Acad. Sci. 98: 31-36.

Lykke-Andersen, J., Shu, M.D., and Steitz, J.A. 2000. Human Upf proteins target an mRNA for nonsense-mediated decay when bound downstream of a termination codon. Cell 103: 1121-1131.

Medghalchi, S.M., Frischmeyer, P.A., Mendell, J.T., Kelly, A.G., Lawler, A.M., and Dietz, H.C. 2001. Rent1, a trans-effector of nonsense-mediated mRNA decay, is essential for mammalian embryonic viability. Hum. Mol. Genet. 10: 99-105.

Mendell, J.T., Sharifi, N.A., Meyers, J.L., Martinez-Murillo, F., and Dietz, H.C. 2004. Nonsense surveillance regulates expression of diverse classes of mammalian transcripts and mutes genomic noise. Nat. Genet. 36: 1073-1078.

Metzstein, M.M. and Krasnow, M.A. 2006. Functions of the nonsense-mediated mRNA decay pathway in Drosophila development. PLoS Genet. 2: e180. doi: 10.1371/journal. pgen.0020180.

Mitrovich, Q.M. and Anderson, P. 2005. mRNA surveillance of expressed pseudogenes in C. elegans. Curr. Biol. 15: $963-$ 967.

Ni, J.Z., Grate, L., Donohue, J.P., Preston, C., Nobida, N., O'Brien, G., Shiue, L., Clark, T.A., Blume, J.E., and Ares Jr., M. 2007. Ultraconserved elements are associated with homeostatic control of splicing regulators by alternative splicing and nonsense-mediated decay. Genes \& Dev. 21: 708718.

Pan, Q., Saltzman, A.L., Kim, Y.K., Misquitta, C., Shai, O., Maquat, L.E., Frey, B.J., and Blencowe, B.J. 2006. Quantitative microarray profiling provides evidence against wide- 


\section{Weischenfeldt et al.}

spread coupling of alternative splicing with nonsense-mediated mRNA decay to control gene expression. Genes \& Dev. 20: $153-158$.

Perlick, H.A., Medghalchi, S.M., Spencer, F.A., Kendzior Jr., R.J., and Dietz, H.C. 1996. Mammalian orthologues of a yeast regulator of nonsense transcript stability. Proc. Nat1. Acad. Sci. 93: 10928-10932.

Pulak, R. and Anderson, P. 1993. mRNA surveillance by the Caenorhabditis elegans smg genes. Genes \& Dev. 7: 18851897.

Rehwinkel, J., Letunic, I., Raes, J., Bork, P., and Izaurralde, E. 2005. Nonsense-mediated mRNA decay factors act in concert to regulate common mRNA targets. RNA 11: 1530-1544.

Reichenbach, P., Hoss, M., Azzalin, C.M., Nabholz, M., Bucher, P., and Lingner, J. 2003. A human homolog of yeast Est1 associates with telomerase and uncaps chromosome ends when overexpressed. Curr. Biol. 13: 568-574.

Ron, D. and Walter, P. 2007. Signal integration in the endoplasmic reticulum unfolded protein response. Nat. Rev. Mol. Cell Biol. 8: 519-529.

Singh, G., Jakob, S., Kleedehn, M.G., and Lykke-Andersen, J. 2007. Communication with the exon-junction complex and activation of nonsense-mediated decay by human Upf proteins occur in the cytoplasm. Mol. Cell 27: 780-792.

Stoilov, P., Daoud, R., Nayler, O., and Stamm, S. 2004. Human tra2- $\beta 1$ autoregulates its protein concentration by influencing alternative splicing of its pre-mRNA. Hum. Mol. Genet. 13: $509-524$

Torrents, D., Suyama, M., Zdobnov, E., and Bork, P. 2003. A genome-wide survey of human pseudogenes. Genome Res. 13: $2559-2567$.

Wang, J., Vock, V.M., Li, S., Olivas, O.R., and Wilkinson, M.F. 2002. A quality control pathway that down-regulates aberrant T-cell receptor (TCR) transcripts by a mechanism requiring UPF2 and translation. J. Biol. Chem. 277: 1848918493.

Weischenfeldt, J., Lykke-Andersen, J., and Porse, B. 2005. Messenger RNA surveillance: Neutralizing natural nonsense. Curr. Biol. 15: R559-R562. doi: 10.1016/j.cub.2005.07.002.

Wittmann, J., Hol, E.M., and Jack, H.M. 2006. hUPF2 silencing identifies physiologic substrates of mammalian nonsensemediated mRNA decay. Mol. Cell. Biol. 26: 1272-1287.

Xing, Y. and Lee, C.J. 2004. Negative selection pressure against premature protein truncation is reduced by alternative splicing and diploidy. Trends Genet. 20: 472-475. 


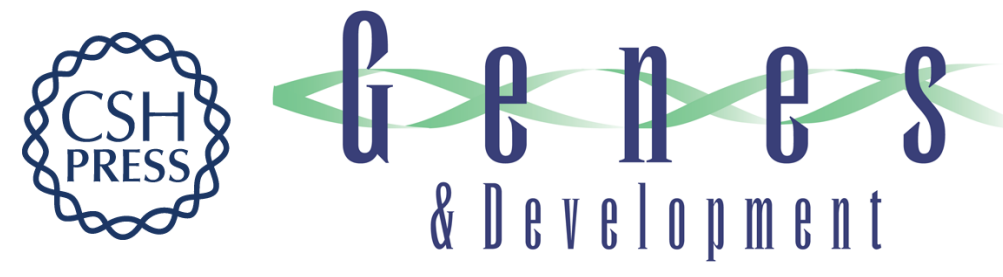

\section{NMD is essential for hematopoietic stem and progenitor cells and for eliminating by-products of programmed DNA rearrangements}

Joachim Weischenfeldt, Inge Damgaard, David Bryder, et al.

Genes Dev. 2008, 22:

Access the most recent version at doi:10.1101/gad.468808

Supplemental http://genesdev.cshlp.org/content/suppl/2008/04/29/22.10.1381.DC1
Material

References This article cites 46 articles, 19 of which can be accessed free at:

http://genesdev.cshlp.org/content/22/10/1381.full.html\#ref-list-1

License

Email Alerting

Receive free email alerts when new articles cite this article - sign up in the box at the top

Service

right corner of the article or click here.

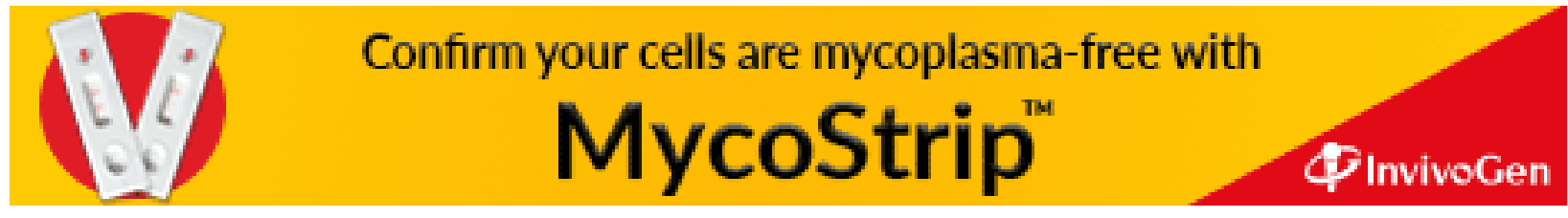

